\title{
A modified approach to the Doplicher/Roberts theorem on the construction of the field algebra and the symmetry group in superselection theory
}

\section{H. Baumgärtel}

Sfb 288 Preprint No. 134

Diese Arbeit ist mit Unterstützung des von der Deutschen Forschungsgemeinschaft getragenen Sonderforschungsbereiches 288 entstanden und als Manuskript vervielfältigt worden.

Berlin, August 1994 



\title{
A modified approach to the Doplicher/Roberts theorem on the construction of the field algebra and the symmetry group in superselection theory
}

\author{
H.Baumgärtel
}

July 22, 1994

\begin{abstract}
Let $\mathcal{A}$ be a unital $C^{*}$-algebra with trivial center $\mathcal{Z}(\mathcal{A})=\mathrm{C} 1$. Let $\mathcal{T}$ denote a tensorial category of unital endomorphisms of $\mathcal{A}$ equipped with several properties to be explained in the text.

Doplicher and Roberts have shown, among other things, that there is a $C^{*}$. algebra $\mathcal{F} \supset \mathcal{A}$ and a compact group $\mathcal{G}$ of automorphisms of $\mathcal{F}$ such that $\mathcal{F}$ is a Hilbert $C^{*}$-system over $\mathcal{A}$ w.r.t. $\mathcal{G}$ where $\mathcal{A}$ is the fixed point algebra w.r.t. $\mathcal{G}, \mathcal{G}=\operatorname{stab} \mathcal{A}$ and the objects $\rho \in \mathrm{Ob} \mathcal{T}$ are characterized as the canonical endomorphisms of certain algebraic $\mathcal{G}$-invariant Hilbert spaces $\mathcal{H}_{\rho} \subset \mathcal{F}$, see Doplicher/Roberts $[1,2,3]$.

The starting point of the approach presented in this paper to point out the mentioned result is an $\mathcal{A}$-leftmodule $\mathcal{F}_{0}:=\left\{\sum_{\rho, j} A_{\rho, j} \Phi_{\rho, j}\right\} . \rho$ runs through a full system of irreducible and mutually disjoint objects of $\mathcal{T}, j=1,2, \ldots, d(\rho)$, where $d(\rho)$ denotes the statistical dimension of $\rho,\left\{\Phi_{\rho j}\right\}_{j=1}^{d(\rho)}$ is an orthonormal basis of a $d(\rho)$-dimensional Hilbert space. The system $\left\{\Phi_{\rho j}\right\}_{\rho j}$ forms a leftmodule basis of $\mathcal{F}_{0}$, the coefficients $A_{\rho j}$ are members of $\mathcal{A}$. The strategy is to equip successively $\mathcal{F}_{0}$ with a bimodule structure, a product and a $*$-structure and finally with a $C^{*}$-norm $\|.\|_{*}$. The symmetry group $\mathcal{G}$ appears as the group of all automorphisms of the *-algebra $\mathcal{F}_{0}$ leaving the $\mathcal{A}$-scalar product $\langle F, G\rangle:=$ $\sum_{\rho, j} d(\rho)^{-1} A_{\rho j} B_{\rho j}^{*}$ invariant, where $F=\sum_{\rho, j} A_{\rho j} \Phi_{\rho j}, G=\sum_{\rho, j} B_{\rho j} \Phi_{\rho j}$. The field algebra is then given by $\mathcal{F}:=\operatorname{clo}_{\|-\| *} \mathcal{F}_{0}$.
\end{abstract}

\section{Introduction}

A few years ago Doplicher and Roberts established a new duality theory for compact groups which is a deepening of the well-known duality thcory of Tannaka/Krein. They developed this theory in connection with the superselection problem of the algebraic quantum field theory (see Doplicher/Roberts $[4,5,1,2,3]$ ). In the algebraic quantum field theory the main part of the superselection problem is to construct an extension $\mathcal{F} \supset \mathcal{A}$ of the quasilocal observable algebra $\mathcal{A}$, called field algebra, which should describe the sector structure of $\mathcal{A}$ and the transition rules by a symmetry principle 
(e.g. a compact group $\mathcal{G}$ ) on $\mathcal{F}$ such that, for example, the fusion rules are explained in group-theoretical terms of $\mathcal{G}$.

In a recent paper [6] (see also Baumgärtel/Wollenberg [7]) the author described a rather simple construction of the field algebra $\mathcal{F}$ as a Hilbert $C^{*}$-system over $\mathcal{A}$ (or a crossed product of $\mathcal{A}$ with a group dual of $\mathcal{G}$ ), if the group $\mathcal{G}$ is known and if an action of a group dual of $\mathcal{G}$ on $\mathcal{A}$ is given a priori.

The present paper is an attempt to use the method of [6] for a construction of $\mathcal{F}$ and of the group $\mathcal{G}$ (which appears as the invariance group of an $\mathcal{A}$-scalar product). An essential point of this ansatz is to construct Hilbert spaces $\mathcal{H}_{\alpha}$ in the $\mathcal{A}$-bimodule $\mathcal{F}_{0}$ to start with, where the Hilbert spaces $\mathcal{H}_{\alpha}$ correspond bijectively to the objects $\alpha$ of the category $\mathcal{T}$ of endomorphisms of $\mathcal{A}$.

This approach shows directly the intrinsic structure of $\mathcal{F}$ and $\mathcal{G}$. On the other hand, it is not (completely) independent of Doplicher/Roberts deep duality theory. At a crucial point in the treatment (introduction of the product structure in $\mathcal{F}_{0}$ ) the author used an important result of Doplicher/Roberts ([2,4.17 Theorem, p.191]) to proceed further.

\section{Preliminaries}

Let $\mathcal{A}$ be a unital $C^{*}$-algebra with center $\mathcal{Z}(\mathcal{A})=\mathrm{C} 1$. By $\rho, \sigma, \ldots$ we denote unital endomorphisms of $\mathcal{A}$. We use the denotation

$$
(\rho, \sigma):=\{T \in \mathcal{A}: T \rho(A)=\sigma(A) T, A \in \mathcal{A}\}
$$

for the linear space of all intertwiners between $\rho$ and $\sigma$. The identical endomorphism is denoted by $\iota$. If $R \in\left(\rho, \rho^{\prime}\right), S \in\left(\sigma, \sigma^{\prime}\right)$ then $R \rho(S)=\rho^{\prime}(S) R \in\left(\rho \sigma, \rho^{\prime} \sigma^{\prime}\right)$. We use the notation $R \times S:=R \rho(S)$. Note that $(\iota, \iota)=\mathbf{C} 1$ and $1 \in(\rho, \rho)$ for all $\rho$, this is indicated by $1=1_{\rho}$.

We consider categories $\mathcal{T}$ of unital endomorphisms equipped with the following properties:

(T1) $\iota \in \mathrm{Ob} \mathcal{T}$.

(T2) If $\rho \in \mathrm{Ob} \mathcal{T}$ and $U \in(\rho, \sigma), U$ unitary, then $\sigma \in \mathrm{Ob} \mathcal{T}$.

(T3) $\rho, \sigma \in \mathrm{Ob} \mathcal{T}$ implies $\rho \sigma \in \mathrm{Ob} \mathcal{T}$.

(T4) The set of arrows between $\rho$ and $\sigma$ is given by $(\rho, \sigma)$.

(T5) If $\rho \in \mathrm{Ob} \mathcal{T}$ and if $0 \neq E \in(\rho, \rho)$ is a selfadjoint projection then there is an isometry $V \in \mathcal{A}, V V^{*}=E$ such that $\sigma \in \mathrm{Ob} \mathcal{T}$ where $\sigma(A):=V^{*} \rho(A) V$, i.e. $\operatorname{Ob} \mathcal{T}$ is closed w.r.t. subobjects.

(T6) If $\rho, \sigma \in \mathrm{Ob} \mathcal{T}$ then there are isometries $V, W \in \mathcal{A}$ with $V V^{*}+W W^{*}=1$ such that $\tau \in \mathrm{Ob} \mathcal{T}$ where $\tau(A):=V \rho(A) V^{*}+W \sigma(A) W^{*}$, i.e. Ob $\mathcal{T}$ is closed w.r.t. direct sums.

(T7) There is a permutation structure on $\mathcal{T}$, i.e. there is an assignment $\{\rho, \sigma\} \rightarrow$ $\epsilon(\rho, \sigma) \in(\rho \sigma, \sigma \rho), \epsilon(\rho, \sigma)$ unitary, with the following properties:

$$
\epsilon(\sigma, \rho) \epsilon(\rho, \sigma)=1_{\rho \sigma},
$$




$$
\begin{gathered}
\epsilon(\iota, \rho)=\epsilon(\rho, \iota)=1_{\rho} \\
\epsilon(\rho \sigma, \tau)=\epsilon(\rho, \tau) \times 1_{\sigma} \cdot 1_{\rho} \times \epsilon(\sigma, \tau) \\
\epsilon\left(\rho^{\prime}, \sigma^{\prime}\right) \cdot R \times S=S \times R \cdot \epsilon(\rho, \sigma), R \in\left(\rho, \rho^{\prime}\right), S \in\left(\sigma, \sigma^{\prime}\right)
\end{gathered}
$$

(T8) There is a conjugation structure on $\mathcal{T}$, i.e. there is an assignment $\mathrm{Ob} \mathcal{T} \ni$ $\rho \rightarrow \bar{\rho} \in \mathrm{Ob} \mathcal{T}$ such that there are corresponding arrows $R_{\rho} \in(\iota, \bar{\rho} \rho), S_{\rho} \in(\iota, \rho \bar{\rho})$ with the following properties:

$$
\begin{gathered}
S_{\rho}:=\epsilon(\bar{\rho}, \rho) R_{\rho}, \\
S_{\rho}^{*} \times 1_{\rho} \cdot 1_{\rho} \times R_{\rho}=1_{\rho}, \\
R_{\rho}^{*} \times 1_{\bar{\rho}} \cdot 1_{\bar{\rho}} \times S_{\rho}=1_{\bar{\rho}} .
\end{gathered}
$$

The endomorphism $\rho$ is called irreducible if $(\rho, \rho)=\mathrm{C} 1 ; \rho$ and $\sigma$ are called disjoint if $(\rho, \sigma)=\{0\}$, they are called unitarily equivalent if there is a unitary $U \in(\rho, \sigma)$.

The theory of $\mathcal{T}$ is well-known, it is developed in Doplicher/Roberts [2,p.164 f.]. We recall several properties: $\bar{\rho}$ is unique up to unitary equivalence, the statistical dimension $d(\rho) \in \mathbf{N}$ given by $d(\rho) 1=R_{\rho}^{*} R_{\rho}$ satisfies $d\left(\rho_{1} \rho_{2}\right)=$ $d\left(\rho_{1}\right) d\left(\rho_{2}\right), d\left(W_{1} \rho_{1}(\cdot) W_{1}^{*}+W_{2} \rho_{2}(\cdot) W_{2}^{*}\right)=d\left(\rho_{1}\right)+d\left(\rho_{2}\right)$. If $\rho_{1}, \rho_{2}$ are unitarily equivalent then $d\left(\rho_{1}\right)=d\left(\rho_{2}\right)$, furthermore $d(\rho)=d(\bar{\rho})$. If $0 \neq E \in(\rho, \rho)$ is a selfadjoint projection then $d\left(V^{*} \rho(\cdot) V\right)=R_{\rho}^{*} \bar{\rho}(E) R_{\rho}$, where $V V^{*}=E$.

Each $\rho$ can be decomposed into a finite direct sum of irreducibles, $\rho(\cdot)=$ $\sum_{j=1}^{r} W_{j} \rho_{j}(\cdot) W_{j}^{*}, \quad W_{j}^{*} W_{k}=\delta_{j k}, \quad \sum_{j} W_{j} W_{j}^{*}=1, \rho_{j}$ irreducible; $(\rho, \rho)$ is finitedimensional. If $\tau$ is irreducible, $\rho$ arbitrary then $(\tau, \rho)$ is a finite-dimensional algebraic Hilbert space in $\mathcal{A}$, for $A, B \in(\tau, \rho)$ the scalar product $(A, B)$ is given by $(A, B) 1=A^{*} B ; \operatorname{dim}(\tau, \rho)=: m(\tau, \rho)$ coincides with the multiplicity of the equivalence class of $\tau$ occurring in the decomposition of $\rho$, i.e.

$$
d(\rho)=\sum_{\tau} m(\tau, \rho) d(\tau)
$$

For later use recall that $\rho \in \mathcal{T}$ is called special if $\operatorname{det} \rho=\iota($ see $[2$, p.174] or $[7$, p.271]).

\section{The $\mathcal{A}$-bimodule $\mathcal{F}_{0}$ and the Hilbert spaces $\mathcal{H}_{\rho}$}

We choose a full system $\hat{T}$ of irreducible and mutually disjoint endomorphisms $\tau \in \mathrm{Ob} \mathcal{T}$. To each $\tau$ we assign a $d(\tau)$-dimensional Hilbert space $\mathcal{H}_{\tau}, \mathcal{H}_{t}:=\mathbf{C} 1$. We choose an orthonormal basis $\Phi_{\tau j}, j=1,2, \ldots, d(\tau)$, of $\mathcal{H}_{\tau}, \Phi_{\imath}:=1$, and we introduce the $\mathcal{A}$-leftmodule

$$
\mathcal{F}_{0}:=\left\{\sum_{\tau, j} A_{\tau, j} \Phi_{\tau j}, A_{\tau j} \in \mathcal{A}, \text { finite sum }\right\}
$$

where $\left\{\Phi_{\tau j}\right\}_{\tau j}$ is assumed to be a leftmodule basis (then each other system $\left\{\Psi_{\tau j}\right\}_{\tau j}$ of orthonormal bases of $\mathcal{H}_{\tau}$ turns out to be a leftmodule basis). Note that $\mathcal{A} \subset \mathcal{F}_{0} . \mathcal{F}_{0}$ is a bimodule w.r.t. the definition

$$
\Phi A=\tau(A) \Phi, \quad \Phi \in \mathcal{H}_{\tau}, A \in \mathcal{A}
$$


and extension by linearity to the whole $\mathcal{F}_{0}$. Then the relative commutant $\mathcal{A}^{\prime} \cap \mathcal{F}_{0}:=$ $\left\{F \in \mathcal{F}_{0}: A F=F A, A \in \mathcal{A}\right\}$ equals $\mathrm{C} 1$.

Let $\rho \in \mathcal{T}$ and $\tau \in \hat{\mathcal{T}}$. We consider the linear subspace spa $\left\{(\tau, \rho) \mathcal{H}_{\tau}\right\} \subset \mathcal{F}_{0}$. Obviously, it is a Hilbert space which can be considered as the tensor product $(\tau, \rho) \otimes$ $\mathcal{H}_{\tau}$ with the scalar product

$$
(A \Phi, B \Psi):=(A, B)_{(\tau, \rho)}(\Phi, \Psi)_{\mathcal{H}_{\tau}}, \quad A, B \in(\tau, \rho) ; \Phi, \Psi \in \mathcal{H}_{\tau}
$$

The sum

$$
\sum_{\tau} \operatorname{spa}\left\{(\tau, \rho) \mathcal{H}_{\tau}\right\}=: \mathcal{H}_{\rho}
$$

is always a direct sum. Therefore $\mathcal{H}_{p}$ is a Hilbert space with the scalar product

$$
\sum_{\tau}\left(X_{\tau}, Y_{\tau}\right), \quad X_{\tau}, Y_{\tau} \in \operatorname{spa}\left\{(\tau, \rho) \mathcal{H}_{\tau}\right\}
$$

The Hilbert spaces $\mathcal{H}_{\tau}$ satisfy the following properties:

\subsection{PROPOSITION. The assignment}

$$
\mathrm{Ob} \mathcal{T} \ni \rho \rightarrow \mathcal{H}_{\rho} \subset \mathcal{F}_{0}
$$

is one-to-one, $X \in \mathcal{H}_{o}$ iff $X A=\rho(A) X$ for all $A \in \mathcal{A}$ and the dimension of $\mathcal{H}_{p}$ coincides with $d(\rho)$,

$$
\operatorname{dim} \mathcal{H}_{\rho}=d(\rho), \quad \rho \in \mathcal{T}
$$

Proof. Let $X \in \mathcal{H}_{\rho}$. Then $X=\sum_{\tau} A_{\tau} \Phi_{\tau}$ with $A_{\tau} \in(\tau, \rho), \Phi_{\tau} \in \mathcal{H}_{\tau}$. Therefore $X A=\sum_{\tau} A_{\tau} \Phi_{\tau} A=\sum_{\tau} A_{\tau} \tau(A) \Phi_{\tau}=\rho(A) \sum_{\tau} A_{\tau} \Phi_{\tau}=\rho(A) X$. Conversely, let $X \in$ $\mathcal{F}_{0}$, i.e. $X=\sum_{\tau, j} A_{\tau j} \Phi_{\tau j}, A_{\tau j} \in \mathcal{A}$ and $X A=\rho(A) X$. This means

$$
\sum_{\tau, j} A_{\tau j} \tau(A) \Phi_{\tau j}=\sum_{\tau, j} \rho(A) A_{\tau j} \Phi_{\tau j}, \quad A \in \mathcal{A},
$$

or

i.e $A_{\tau j} \in(\tau, \rho)$.

$$
A_{\tau j} \tau(A)=\rho(A) A_{\tau j}, \quad A \in \mathcal{A},
$$

If $\mathcal{H}_{\rho}=\mathcal{H}_{\sigma}=: \mathcal{H}$ then $\rho(A) X=\sigma(A) X$ follows for all $A \in \mathcal{A}, X \in \mathcal{H}$, in particular for $X \in \mathcal{H}_{r}$ where $(\tau, \rho) \neq\{0\}$, i.e. $\rho(A) \Phi_{\tau j}=\sigma(A) \Phi_{\tau j}$ or $\rho(A)=\sigma(A)$ for all $A \in \mathcal{A}$, i.e. $\rho=\sigma$. Finally, we have

$$
\begin{gathered}
\operatorname{dim} \mathcal{H}_{p}=\sum_{\tau} \operatorname{dim}\left(\operatorname{spa}\left\{(\tau, \rho) \mathcal{H}_{\tau}\right\}\right)=\sum_{\tau} \operatorname{dim}(\tau, \rho) \operatorname{dim} \mathcal{H}_{\tau}= \\
=\sum_{\tau} m(\tau, \rho) d(\tau)=d(\rho) . \square
\end{gathered}
$$

The arrows from $(\rho . \sigma)$ can be interpreted as linear operators from $\mathcal{L}\left(\mathcal{H}_{\rho} \rightarrow \mathcal{H}_{\sigma}\right)$ by the assignment

$$
(\rho, \sigma) \ni A \rightarrow \phi(A) \in \mathcal{L}\left(\mathcal{H}_{\rho} \rightarrow \mathcal{H}_{\sigma}\right): \phi(A) X:=A X, \quad X \in \mathcal{H}_{\rho}
$$


Note that the assignment $(2)$ is injective and that $\phi\left(A^{*}\right)$ is the Hilbert space adjoint of $\phi(A)$ :

3.2.PROPOSITION. Let $A_{1}, A_{2} \in(\rho, \sigma)$. If $\phi\left(A_{1}\right)=\phi\left(A_{2}\right)$ then $A_{1}=A_{2}$. Furthermore,

$$
\phi\left(A^{*}\right)=\phi(A)^{*}, \quad A \in(\rho, \sigma) .
$$

Proof. According to the assumption we have $A_{1} X=A_{2} X$ for all $X \in \mathcal{H}_{\rho}$. This implies $A_{1} Y=A_{2} Y$ for all $Y \in(\tau, \rho), \tau \in \hat{\mathcal{T}}$, i.e. $\left(A_{1}-A_{2}\right) Y=0$. Choose an orthonormal basis $W_{\tau j} \in(\tau, \rho), j=1,2, \ldots, m(\tau, \rho)$. Then $\sum_{j} W_{\tau j} W_{\tau j}^{*}=E_{\tau} \epsilon$ $(\rho, \rho)$, where $E_{\tau}$ is the central projection assigned to $\tau$. Recall that $\sum_{\tau} E_{\tau}=1$. So first we obtain $\left(A_{1}-A_{2}\right) E_{\tau}=0$ for all $\tau$ occurring in the decomposition of $\rho$ and second $A_{1}-A_{2}=0$. Furthermore, with $A \in(\rho, \sigma), A_{1} \in(\tau, \sigma), A_{2} \in(\tau, \rho)$ we have $\left(A_{1}, A A_{2}\right)_{(\tau, \sigma)}=A_{1}^{*} A A_{2}=\left(A^{*} A_{1}\right)^{*} A_{2}=\left(A^{*} A_{1}, A_{2}\right)_{(\tau, \rho)}$.

\section{Construction of special isometries (cocycles) $J(\rho, \sigma): \mathcal{H}_{\rho} \otimes \mathcal{H}_{\sigma} \rightarrow \mathcal{H}_{\rho \sigma}$ and the product struc- ture in $\mathcal{F}_{0}$}

From (1) and $d(\rho \sigma)=d(\rho) d(\sigma)$ we get immediately $\operatorname{dim} \mathcal{H}_{\rho} \otimes \mathcal{H}_{\sigma}=\operatorname{dim} \mathcal{H}_{\rho \sigma}$, i.e. the Hilbert spaces $\mathcal{H}_{\rho} \otimes \mathcal{H}_{\sigma}$ and $\mathcal{H}_{\rho \sigma}$ are isomorphic. Within the set of all isometries from $\mathcal{H}_{\rho} \otimes \mathcal{H}_{\sigma}$ onto $\mathcal{H}_{\rho \sigma}$ we construct special ones (cocycles) $J(\rho, \sigma)$, equipped with the following properties:

(C1) $J(\rho, \iota)=J(\iota, \rho)=\mathrm{id}_{\mathcal{H}_{\rho}}$,

$(\mathrm{C} 2) J(\rho \sigma, \tau)\left(J(\rho, \sigma) \otimes 1_{\mathcal{H}_{\tau}}\right)=J(\rho, \sigma \tau)\left(1_{\mathcal{H}_{\rho}} \Theta J(\sigma, \tau)\right)$,

(C3) $J\left(\rho^{\prime}, \sigma^{\prime}\right) \phi(A) \otimes \phi(B)=\phi(A \times B) J(\rho, \sigma) . \quad A \in\left(\rho, \rho^{\prime}\right), B \in\left(\sigma, \sigma^{\prime}\right)$,

(C4) $\phi(\epsilon(\rho, \sigma)) J(\rho, \sigma)=J(\sigma, \rho) \Theta\left(\mathcal{H}_{\rho}, \mathcal{H}_{\sigma}\right)$,

where $\Theta\left(\mathcal{H}_{\rho}, \mathcal{H}_{\sigma}\right)$ denotes the flip isomorphism $\mathcal{H}_{\rho} \otimes \mathcal{H}_{\sigma} \rightarrow \mathcal{H}_{\sigma} \otimes \mathcal{H}_{\rho}$, and where $\epsilon(\cdot, \cdot)$ are the unitaries of the permutation structure in $\mathcal{T}$.

Note that one can introduce an equivalence relation within the set of all isometries $J(\rho, \sigma)$ satisfying $(\mathrm{C} 1)-(\mathrm{C} 3)$ by

$$
J^{\prime} \sim J \quad \text { if } \quad J^{\prime}(\rho, \sigma)=V(\rho \sigma)^{-1} J(\rho, \sigma) V(\rho) \otimes V(\sigma)
$$

where $\rho \rightarrow V(\rho) \in \mathcal{L}\left(\mathcal{H}_{\rho}\right)$ is a unitary with the property

$$
\phi(A) V(\rho)=V(\sigma) \phi(A), \quad A \in(\rho, \sigma) .
$$

It turns out that $J(\sigma, \rho) \Theta\left(\mathcal{H}_{\rho}, \mathcal{H}_{\sigma}\right) J(\rho, \sigma)^{-1}$ is an invariant of the equivalence classes and this invariant satisfies the property (T7) of the permutation structure.

We focus on the construction of $J(\rho, \sigma)$ if $\epsilon(\cdot, \cdot)$ is given. We separate three parts of the construction problem:

First we choose a special $\rho \in \mathcal{T}$, we consider the subcategory consisting of all powers $\rho^{n}, n=0,1,2, \ldots$ and we construct $J\left(\rho^{n}, \rho^{m}\right), n, m=0,1,2, \ldots$. At this first stage we use an important result of Doplicher/Roberts [2,4.17 Theorem, p.191]. 
Second we construct corresponding isometries $J(\alpha, \beta)$ for all $\alpha, \beta \in \mathcal{T}$ which are dominated by a special (fixed) $\rho$, i.e. $\alpha, \beta$ are assumed to be subobjects of direct sums of powers of $\rho$.

Third, using the second step, we construct a unique function $\mathcal{T} \times \mathcal{T} \ni\{\alpha, \beta\} \rightarrow$ $J(\alpha, \beta) \in \mathcal{L}\left(\mathcal{H}_{\alpha} \otimes \mathcal{H}_{\beta} \rightarrow \mathcal{H}_{\alpha \beta}\right)$ satisfying $(\mathrm{C} 1)$-(C4)

The first step: Let $\rho \in \mathcal{T}$ be special. We look for auxiliary isometries $\Phi_{n}$ from $\mathcal{H}_{\rho^{n}}$ onto $\mathcal{H}_{\rho}^{n}$, equipped with several properties, where $\mathcal{H}_{\rho}^{n}$ denotes the $\mathrm{n}$-fold tensor product of $\mathcal{H}_{\rho}$.

4.1.PROPOSITION. Let $\rho \in \mathcal{T}$ be special. Then there is a system $\left\{\Phi_{r}\right\}_{r=1}^{\infty}$ of isometries $\Phi_{r}: \mathcal{H}_{\rho^{r}} \rightarrow \mathcal{H}_{\rho}^{r}$ such that the isomorphisms $\beta_{r}^{s}: \mathcal{L}\left(\mathcal{H}_{\rho^{r}} \rightarrow \mathcal{H}_{\rho^{s}}\right) \rightarrow$ $\mathcal{L}\left(\mathcal{H}_{\rho}^{\tau} \rightarrow \mathcal{H}_{\rho}^{s}\right)$ given by

$$
\beta_{r}^{s}(X):=\Phi_{s} \circ X \circ \Phi_{r}^{-1}, \quad X \in \mathcal{L}\left(\mathcal{H}_{\rho^{r}} \rightarrow \mathcal{H}_{\rho^{s}}\right),
$$

satisfies

$$
\left(\beta_{r+s}^{r^{\prime}+s^{\prime}} \circ \phi\right)(A \times B)=\left(\beta_{r}^{r^{\prime}} \circ \phi\right)(A) \otimes\left(\beta_{s}^{s^{\prime}} \circ \phi\right)(B)
$$

for all $A \in\left(\rho^{r}, \rho^{r^{\prime}}\right), B \in\left(\rho^{s}, \rho^{s^{\prime}}\right)$. Moreover,

$$
\left(\beta_{n}^{n} \circ \phi\right)\left(\epsilon_{\rho}^{(n)}(\pi)\right)=\Theta_{\rho}^{(n)}(\pi), \quad \pi \in \mathbf{P}_{n}, n=1,2, \ldots
$$

holds, i.e.

$$
\Phi_{n} \circ \phi\left(\epsilon_{p}^{(n)}(\pi)\right)=\Theta_{\rho}^{(n)}(\pi) \circ \Phi_{n}, \quad \pi \in \mathbf{P}_{n} .
$$

Proof. According to Doplicher/Roberts [2,4.17 Theorem, p.191] there is a closed subgroup $\mathcal{G}$ of $\operatorname{SU}\left(\mathcal{H}_{p}\right)$ and a unital monomorphism $\alpha$ of a graded $C^{*}$-algebra $C^{*}(\rho)$, constructed by the intertwiner spaces $\left(\rho^{r}, \rho^{s}\right)$, which maps $C^{*}(\rho)$ into the Cuntz algebra $\mathcal{C}_{\mathcal{H}_{\rho}}$ generated by $\mathcal{H}_{\rho}$. One has $\alpha\left(C^{*}(\rho)\right)=\mathcal{C}_{\mathcal{G}}$ where $\mathcal{C}_{\mathcal{G}}$ denotes the fixed point algebra of $\mathcal{C}_{\mathcal{H}_{\rho}}$ under the canonical action of $\mathcal{G}$ and

$$
\alpha \mid\left(\rho^{n}, \rho^{m}\right)=\mathcal{L}_{\mathcal{G}}\left(\mathcal{H}_{\rho}^{n} \rightarrow \mathcal{H}_{\rho}^{m}\right)
$$

Note that $\mathcal{L}\left(\mathcal{H}_{\rho}^{n} \rightarrow \mathcal{H}_{\rho}^{m}\right)$ is interpreted, as usual, as a subspace of $C_{\mathcal{H}_{\rho}}$ and $\mathcal{L}_{\mathcal{G}}\left(\mathcal{H}_{\rho}^{n} \rightarrow\right.$ $\left.\mathcal{H}_{\rho}^{m}\right)$ denotes the intersection of $\mathcal{C}_{\mathcal{G}}$ and $\mathcal{L}\left(\mathcal{H}_{\rho}^{n} \rightarrow \mathcal{H}_{\rho}^{m}\right)$, i.e. the set of all intertwiners w.r.t. the (tensorial) action of $\mathcal{G}$ on $\mathcal{H}_{\rho}^{n}$ resp. $\mathcal{H}_{\rho}^{m}$. Note further that $\alpha$ satisfies the relations

$$
\alpha(A \times B)=\alpha(A) \otimes \alpha(B), \quad A \in\left(\rho^{r}, \rho^{r^{\prime}}\right), B \in\left(\rho^{s}, \rho^{s^{\prime}}\right),
$$

and

$$
\alpha\left(\epsilon_{\rho}(\pi)\right)=\Theta_{\mathcal{H}_{\rho}}(\pi), \quad \pi \in \mathbf{P}_{\infty}
$$

Now we form the Fock spaces

$$
\mathcal{F}:=\oplus_{n=0}^{\infty} \mathcal{H}_{\rho^{n}}, \mathcal{H}_{\rho}^{0}:=\mathbf{C} ; \mathcal{F}_{1}:=\oplus_{n=0}^{\infty} \mathcal{H}_{\rho}^{n}, \mathcal{H}_{\rho}^{0}:=\mathbf{C} \text {, (Hilbert sums) }
$$

By $E_{n}$ we denote the projection of $\mathcal{F}$ onto its component $\mathcal{H}_{\rho^{n}}$; correspondingly $F_{n}$ is the projection of $\mathcal{F}_{1}$ onto its component $\mathcal{H}_{\rho}^{n}$.

We consider the v.Neumann algebras $\mathcal{M}, \mathcal{M}_{1}$ on $\mathcal{F}$ resp. $\mathcal{F}_{1}$, generated by $\phi\left(\left(\rho^{n}, \rho^{m}\right)\right) \subset \mathcal{L}\left(\mathcal{H}_{\rho^{n}} \rightarrow \mathcal{H}_{\rho^{m}}\right)$ resp. by $\mathcal{L}_{\mathcal{G}}\left(\mathcal{H}_{\rho}^{n} \rightarrow \mathcal{H}_{\rho}^{m}\right)$, i.e.

$$
\mathcal{M}:=\left(\bigcup_{n, m=0}^{\infty} E_{m} \phi\left(\left(\rho^{n}, \rho^{m}\right)\right) E_{n}\right)^{\prime \prime},
$$




$$
\mathcal{M}_{1}:=\left(\bigcup_{n, m=0}^{\infty} F_{m} \mathcal{L}_{\mathcal{G}}\left(\mathcal{H}_{\rho}^{n} \rightarrow \mathcal{H}_{\rho}^{m}\right) F_{n}\right)^{\prime \prime}
$$

Then the Doplicher/Roberts-isomorphism $\alpha$ induces an isomorphism $\beta$ between the algebras $\mathcal{M}$ and $\mathcal{M}_{1}, \beta: \mathcal{M} \rightarrow \mathcal{M}_{1}$, where

$$
\beta\left(E_{m} \phi(A) E_{n}\right)=F_{m} \alpha(A) F_{n}, \quad A \in\left(\rho^{n}, \rho^{m}\right),
$$

(see for example Kadison/Ringrose [9, p.427]). It has the property

$$
\beta\left(E_{m} X E_{n}\right)=F_{m} \beta(X) F_{n}, \quad X \in \mathcal{M} .
$$

Obviously, $\mathcal{M}_{1}=U(\mathcal{G})^{\prime}$ where $U(\mathcal{G})$ denotes the canonical representation of $\mathcal{G}$ on $\mathcal{F}_{1}$ given by the identical representation on $\mathcal{H}_{\rho}$ : Note that $\mathcal{F}_{1} \ni X=\sum_{n, m=0}^{\infty} F_{m} X F_{n}$ (strong convergence), hence $X \in U(\mathcal{G})^{\prime}$ implies $F_{m} X F_{n} U(g)=U(g) F_{m} X F_{n}$ because $F_{n} U(g)=U(g) F_{n}$, for all $g \in \mathcal{G}$. That is, we have $F_{m} X F_{n} \mid \mathcal{H}_{\rho}^{n} \in \mathcal{L}_{\mathcal{G}}\left(\mathcal{H}_{\rho}^{n} \rightarrow \mathcal{H}_{\rho}^{m}\right)$ or $F_{m} X F_{n}=F_{m} Y F_{n}$ with $Y \in \mathcal{L}_{\mathcal{G}}\left(\mathcal{H}_{\rho}^{n} \rightarrow \mathcal{H}_{\rho}^{m}\right)$. The other inclusion is trivial.

Thus $\mathcal{M}_{1}$ is of type $I$, therefore, as is well-known (see e.g. $[9$, p.660ff.]) the isomorphism $\beta$ is spatial, i.e. there is an isometry $\Phi: \mathcal{F} \rightarrow \mathcal{F}_{1}$ such that $\beta(X)=$ $\Phi X \Phi^{-1}, X \in \mathcal{M}$. Now we have $\beta\left(E_{n}\right)=F_{n} \beta\left(E_{n}\right) F_{n}$, i.e. $F_{n} \beta\left(E_{n}\right)=\beta\left(E_{n}\right) F_{n}=$ $\beta\left(E_{n}\right)$ or

$$
F_{n} \cdot \Phi E_{n} \Phi^{-1}=\Phi E_{n} \Phi^{-1} \cdot F_{n}=\Phi E_{n} \Phi^{-1}
$$

Since $\operatorname{dim} \Phi E_{n} \Phi^{-1}=\operatorname{dim} E_{n}=\operatorname{dim} F_{n}$ we obtain $F_{n}=\Phi E_{n} \Phi^{-1}$ or $\Phi E_{n}=F_{n} \Phi$. This means that $\Phi$ is diagonal,

$$
\Phi=\operatorname{diag}\left\{\Phi_{n}\right\}_{n=0}^{\infty}
$$

where $\Phi_{n}: \mathcal{H}_{\rho^{n}} \rightarrow \mathcal{H}_{\rho}^{n}, n=0,1,2, \ldots$, is isometric.

Therefore, the isomorphisms

$$
\beta_{n}^{m}:=\Phi_{m} \phi(A) \Phi_{n}^{-1}, \quad A \in\left(\rho^{n}, \rho^{m}\right)
$$

satisfy the equation

$$
\beta_{n}^{m}(\phi(A))=\alpha(A), \quad A \in\left(\rho^{n}, \rho^{m}\right),
$$

Now from (7) we obtain (5). Furthermore, from (6) we obtain (4): Namely, $A \in\left(\rho^{r}, \rho^{r^{\prime}}\right), B \in\left(\rho^{s}, \rho^{s^{\prime}}\right)$ implies $A \times B \in\left(\rho^{r+s}, \rho^{r^{\prime}+s^{\prime}}\right)$, i.e. we have $\phi(A) \in$ $\mathcal{L}\left(\mathcal{H}_{\rho^{r}} \rightarrow \mathcal{H}_{\rho^{r^{\prime}}}\right), \phi(B) \in \mathcal{L}\left(\mathcal{H}_{\rho^{s}} \rightarrow \mathcal{H}_{\rho^{s^{\prime}}}, \phi(A \times B) \in \mathcal{L}\left(\mathcal{H}_{\rho^{r+s}} \rightarrow \mathcal{H}_{\rho^{r^{\prime}+\varepsilon^{\prime}}}\right)\right.$, i.e.

$$
\alpha(A \times B)=\beta_{r+s}^{r^{\prime}+s^{\prime}}(\phi(A \times B))
$$

and

$$
\alpha(A) \otimes \alpha(B)=\beta_{r}^{r^{\prime}}(\phi(A)) \otimes \beta_{s}^{s^{\prime}}(\phi(B)) .
$$

It would be nice to have a proof for the existence of the $\beta_{n}^{m}$ which is independent of the mentioned theorem of Doplicher/Roberts.

Using the isometries $\Phi_{n}$, we define isometries from $\mathcal{H}_{\rho^{r}} \otimes \mathcal{H}_{\rho^{s}}$ onto $\mathcal{H}_{\rho^{r+s}}$ by

$$
J\left(\rho^{r}, \rho^{s}\right):=\Phi_{r+s}\left(\Phi_{r} \otimes \Phi_{s}\right)^{-1} .
$$

Then we obtain 
4.2.PROPOSITION. The isometries $J\left(\rho^{r}, \rho^{s}\right)$ satisfy $(\mathrm{C} 1)-(\mathrm{C} 4)$.

Proof. (C1) is obvious. (C2): We have to prove

$$
J\left(\rho^{r+n}, \rho^{s}\right)\left(J\left(\rho^{r}, \rho^{n}\right) \otimes 1_{\mathcal{H}_{\rho^{s}}}\right)=J\left(\rho^{r}, \rho^{n+s}\right)\left(1_{\mathcal{H}_{\rho^{r}}} \otimes J\left(\rho^{n}, \rho^{s}\right)\right) .
$$

By the definition of $J(\cdot, \cdot)$ we have

$$
\Phi_{r+n+s}=J\left(\rho^{r+n}, \rho^{s}\right) \Phi_{r+n} \otimes \Phi_{s}=J\left(\rho^{r}, \rho^{n+s}\right)\left(\Phi_{r} \otimes \Phi_{n+s}\right)
$$

and

$$
\begin{gathered}
J\left(\rho^{r+n}, \rho^{s}\right) \Phi_{r+n} \otimes \Phi_{s}=J\left(\rho^{r+n}, \rho^{s}\right)\left(\left\{J\left(\rho^{r}, \rho^{n}\right)\left(\Phi_{r} \otimes \Phi_{n}\right)\right\} \otimes \Phi_{s}\right)= \\
=J\left(\rho^{r+n}, \rho^{s}\right)\left(J\left(\rho^{r}, \rho^{n}\right) \otimes 1_{\mathcal{H}_{\rho^{s}}}\right)\left(\Phi_{r} \otimes \Phi_{n} \otimes \Phi_{s}\right) .
\end{gathered}
$$

For the right hand side of $(8)$ we get similarly

$J\left(\rho^{r}, \rho^{n+s}\right)\left(\Phi_{r} \otimes\left\{J\left(\rho^{n}, \rho^{s}\right)\left(\Phi_{n} \otimes \Phi_{s}\right)\right\}\right)=J\left(\rho^{r}, \rho^{n+s}\right)\left(1_{\mathcal{H}_{\rho^{r}}} \otimes J\left(\rho^{n}, \rho^{s}\right)\right)\left(\Phi_{r} \otimes \Phi_{n} \otimes \Phi_{s}\right)$.

(C3): For brevity we write simply $\beta$ instead of $\beta_{r}^{r^{\prime}}$ etc. For $A \in\left(\rho^{r}, \rho^{r^{\prime}}\right), B \in\left(\rho^{s}, \rho^{s^{\prime}}\right)$ we have

$$
\begin{gathered}
J\left(\rho^{r^{\prime}}, \rho^{s^{\prime}}\right) \phi(A) \otimes \phi(B)=\Phi_{r^{\prime}+s^{\prime}}\left\{\left(\Phi_{r^{\prime}}^{-1} \phi(A)\right) \otimes\left(\Phi_{s^{\prime}}^{-1} \phi(B)\right)\right\}= \\
=\Phi_{r^{\prime}+s^{\prime}}\left\{(\beta \circ \phi)(A) \Phi_{r}^{-1} \otimes(\beta \circ \phi)(B) \Phi_{s}^{-1}\right\}=\Phi_{r^{\prime}+s^{\prime}}((\beta \circ \phi)(A) \otimes(\beta \circ \phi)(B)) \Phi_{r}^{-1} \otimes \Phi_{s}^{-1}= \\
=\Phi_{r^{\prime}+s^{\prime}}\left((\beta \circ \phi)(A \times B) \Phi_{r}^{-1} \otimes \Phi_{s}^{-1}\right)=\phi(A \times B) \Phi_{r+s}\left(\Phi_{r}^{-1} \otimes \Phi_{s}^{-1}\right)=\phi(A \times B) J\left(\rho^{r}, \rho^{s}\right) .
\end{gathered}
$$

Finally we prove (C4): We calculate

$$
\begin{gathered}
J\left(\rho^{s}, \rho^{r}\right) \Theta\left(\mathcal{H}_{\rho^{r}}, \mathcal{H}_{\rho^{s}}\right)=\Phi_{r+s}\left(\Phi_{s}^{-1} \otimes \Phi_{r}^{-1}\right) \Theta\left(\mathcal{H}_{\rho^{r}}, \mathcal{H}_{\rho^{s}}\right)=\Phi_{r+s} \Theta\left(\mathcal{H}_{\rho}^{r}, \mathcal{H}_{\rho}^{s}\right)\left(\Phi_{r}^{-1} \otimes \Phi_{s}^{-1}\right)= \\
=\Phi_{r+s} \Theta_{\mathcal{H}_{\rho}}^{(r+s)}((r, s))\left(\Phi_{r}^{-1} \otimes \Phi_{s}^{-1}\right)=\phi\left(\epsilon_{\rho}^{(r+s)}((r, s))\right) \Phi_{r+s}\left(\Phi_{r}^{-1} \otimes \Phi_{s}^{-1}\right)= \\
=\phi\left(\epsilon\left(\rho^{r}, \rho^{s}\right)\right) J\left(\rho^{r}, \rho^{s}\right) . \square
\end{gathered}
$$

The second step: We have to construct $J(\alpha, \beta)$ for direct sums $\alpha, \beta$ of powers of $\rho$ (where $\rho$ is special) and then we have to verify the properties (C1)-(C4). The same procedure is to establish for subobjects of such direct sums. We begin with direct sums: According to their definition we have the following formulas for direct sums $\alpha, \beta, \gamma$, where the isometries $W_{j}, V_{k}, U_{l}$ have the properties mentioned above:

$$
\begin{gathered}
\alpha(\cdot):=\sum_{j} W_{j} \rho^{m_{\jmath}}(\cdot) W_{j}^{*}, \beta(\cdot):=\sum_{k} V_{k} \rho^{n_{k}}(\cdot) V_{k}^{*}, \gamma(\cdot):=\sum_{l} U_{l} \rho^{r_{l}}(\cdot) U_{l}^{*}, \\
W_{j} \in\left(\rho^{m_{\jmath}}, \alpha\right), \quad V_{k} \in\left(\rho^{n_{k}} . \beta\right), \quad U_{l} \in\left(\rho^{r_{l}}, \gamma\right), \\
\mathcal{H}_{\alpha}=\sum_{j} W_{j} \mathcal{H}_{\rho^{m_{\jmath}}}, \quad \mathcal{H}_{\beta}=\sum_{k} V_{k} \mathcal{H}_{\rho^{n_{k}}}, \quad \mathcal{H}_{\gamma}=\sum_{l} U_{l} \mathcal{H}_{\rho^{r_{l}}} .
\end{gathered}
$$

We put

$$
J(\alpha, \beta):=\sum_{j, k} \phi\left(W_{j} \times V_{k}\right) J\left(\rho^{m}, \rho^{n_{k}}\right) \phi\left(W_{j}^{*}\right) \otimes \phi\left(V_{k}^{*}\right)
$$

similarly for the other objects $J(\beta, \gamma), J(\alpha \beta, \gamma)$ etc. Then we assert 
4.3.LEMMA. The isometries (9) satisfy the properties (C1)-(C4).

Proof. (C1) is obvious. (C3): We have

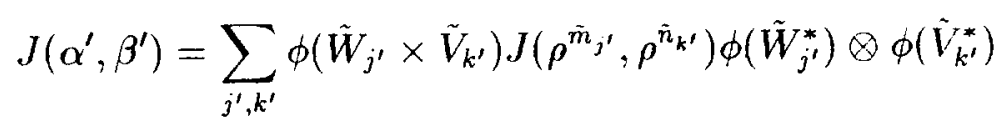

where $\tilde{W}_{j^{\prime}} \in\left(\rho^{\tilde{m}_{j^{\prime}}}, \alpha^{\prime}\right), \tilde{V}_{k^{\prime}} \in\left(\rho^{\tilde{n}_{k^{\prime}}}, \beta^{\prime}\right)$. Let $A \in\left(\alpha, \alpha^{\prime}\right), B \in\left(\beta, \beta^{\prime}\right)$. Then

$$
\tilde{W}_{j^{\prime}}^{*} A W_{j} \in\left(\rho^{m_{\jmath}}, \rho^{\dot{m}_{j^{\prime}}}\right), \quad \tilde{V}_{k^{\prime}}^{*} B V_{k} \in\left(\rho^{n_{k}}, \rho^{\tilde{n}_{k^{\prime}}}\right),
$$

and we have, according to (C3) for the powers of $\rho$

$$
J\left(\rho^{\tilde{m}_{j^{\prime}}}, \rho^{\tilde{n}_{k^{\prime}}}\right) \phi\left(\tilde{W}_{j^{\prime}}^{*} A W_{j}\right) \otimes \phi\left(\tilde{V}_{k^{\prime}}^{*} B V_{k}\right)=\phi\left(\tilde{W}_{j^{\prime}}^{*} A W_{j} \times \tilde{V}_{k^{\prime}}^{*} B V_{k}\right) J\left(\rho^{m_{j}}, \rho^{n_{k}}\right) .
$$

Now multiplying this equation from the left by $\phi\left(\tilde{W}_{j^{\prime}} \times \tilde{V}_{k^{\prime}}\right)$, from the right by $\phi\left(W_{j}^{*}\right) \otimes$ $\phi\left(V_{k}^{*}\right)$ and summing up w.r.t. $j^{\prime}, k^{\prime}, j, k$, we obtain

$$
\begin{gathered}
\sum_{j^{\prime}, k^{\prime}} \phi\left(\tilde{W}_{j^{\prime}} \times \tilde{V}_{k^{\prime}}\right) J\left(\rho^{\tilde{m}_{j^{\prime}}}, \rho^{\tilde{n}_{k^{\prime}}}\right) \phi\left(\tilde{W}_{j^{\prime}}^{*}\right) \otimes \phi\left(\tilde{V}_{k^{\prime}}^{*}\right) \cdot \phi(A) \otimes \phi(B)= \\
=\phi(A \times B) \sum_{j, k} \phi\left(W_{j} \times V_{k}\right) J\left(\rho^{m}, \rho^{n_{k}}\right) \phi\left(W_{j}^{*}\right) \otimes \phi\left(V_{k}^{*}\right)
\end{gathered}
$$

or

$$
J\left(\alpha^{\prime}, \beta^{\prime}\right) \phi(A) \otimes \phi(B)=\phi(A \times B) J(\alpha, \beta) .
$$

(C2): Note that

$$
\alpha \beta(\cdot)=\sum_{j, k} W_{j} \times V_{k} \rho^{m_{j}+n_{k}}(\cdot)\left(W_{j} \times V_{k}\right)^{*}
$$

Then

$$
J(\alpha \beta, \gamma)=\sum_{j, k, l} \phi\left(\left(W_{j} \times V_{k}\right) \times U_{l}\right) J\left(\rho^{m_{j}+n_{k}}, \rho^{r_{l}}\right) \phi\left(\left(W_{j} \times V_{k}\right)^{*}\right) \otimes \phi\left(U_{l}^{*}\right)
$$

and we have to calculate

$$
\begin{aligned}
& J(\alpha \beta, \gamma)\left(J(\alpha, \beta) \otimes 1_{\mathcal{H}_{\gamma}}\right)= \\
& \sum_{j, k, l} \phi\left(W_{j} \times V_{k} \times U_{l}\right) J\left(\rho^{m_{j}+n_{k}}, \rho^{r_{l}}\right) \phi\left(W_{j} \times V_{k}\right)^{*} \otimes \phi\left(U_{l}\right)^{*} \\
& \cdot \sum_{j^{\prime}, k^{\prime}} \phi\left(W_{j^{\prime}} \times V_{k^{\prime}}\right) J\left(\rho^{m_{j^{\prime}}}, \rho^{n_{k^{\prime}}}\right)\left(\phi\left(W_{j^{\prime}}\right)^{*} \otimes \phi\left(V_{k^{\prime}}\right)^{*}\right) \otimes 1_{\mathcal{H}_{\gamma}} \\
& =\sum_{j, k, l} \sum_{j^{\prime}, k^{\prime}} \phi\left(W_{j} \times V_{k} \times U_{l}\right) J\left(\rho^{m_{j}+n_{k}}, \rho^{r_{l}}\right) .
\end{aligned}
$$

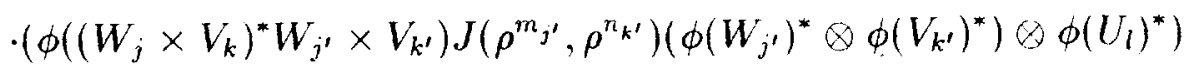

$$
\begin{aligned}
& =\sum_{j, k, l} \phi\left(W_{j} \times V_{k} \times U_{l}\right) J\left(\rho^{m_{\jmath}+n_{k}}, \rho^{r_{l}}\right)\left(J\left(\rho^{m_{\jmath}}, \rho^{n_{k}}\right) \otimes 1_{\mathcal{H}_{\rho^{r_{l}}}}\right)\left(\phi\left(W_{j}\right)^{*} \otimes \phi\left(V_{k}\right)^{*} \otimes \phi\left(U_{l}\right)^{*}\right) .
\end{aligned}
$$


On the other hand,

$$
\begin{gathered}
J(\alpha, \beta \gamma)\left(1_{\mathcal{H}_{a}} \otimes J(\beta, \gamma)\right)= \\
\sum_{j, k, l} \phi\left(W_{j} \times V_{k} \times U_{l}\right) J\left(\rho^{m,}, \rho^{n_{k}+r_{l}}\right)\left(1_{\mathcal{H}_{\rho^{m_{j}}}} \otimes J\left(\rho^{n_{k}}, \rho^{r_{l}}\right)\right)\left(\phi\left(W_{j}\right)^{*} \otimes \phi\left(V_{k}\right)^{*} \otimes \phi\left(U_{l}\right)^{*}\right) .
\end{gathered}
$$

Hence the validity of (C2) for powers of $\rho$ implies its validity for direct sums. (C4): We assert that

$$
J(\beta, \alpha) \Theta\left(\mathcal{H}_{\alpha}, \mathcal{H}_{\beta}\right)=\phi(\epsilon(\alpha, \beta)) J(\alpha, \beta) .
$$

We calculate the left hand side: it equals

$$
\begin{gathered}
\sum_{k, j} \phi\left(V_{k} \times W_{j}\right) J\left(\rho^{n_{k}}, \rho^{m_{j}}\right) \phi\left(V_{k}\right)^{*} \otimes \phi\left(W_{j}\right)^{*} \Theta\left(\mathcal{H}_{\alpha}, \mathcal{H}_{\beta}\right)= \\
=\sum_{k, j} \phi\left(V_{k} \times W_{j}\right) J\left(\rho^{n_{k}}, \rho^{m_{j}}\right) \Theta\left(\mathcal{H}_{\rho^{m_{j}}}, \mathcal{H}_{\rho^{n_{k}}}\right) \phi\left(W_{j}\right)^{*} \otimes \phi\left(V_{k}\right)^{*} \\
=\sum_{k, j} \phi\left(V_{k} \times W_{j}\right) \phi\left(\epsilon\left(\rho^{m_{j}}, \rho^{n_{k}}\right)\right) J\left(\rho^{m_{j}}, \rho^{n_{k}}\right) \phi\left(W_{j}\right)^{*} \otimes \phi\left(V_{k}\right)^{*} \\
=\sum_{k, j} \phi(\epsilon(\alpha, \beta)) \phi\left(W_{j} \times V_{k}\right) J\left(\rho^{m_{j}}, \rho^{n_{k}}\right) \phi\left(W_{j}\right)^{*} \otimes \phi\left(V_{k}\right)^{*}=\phi(\epsilon(\alpha, \beta)) J(\alpha, \beta),
\end{gathered}
$$

i.e. (10) is proved. $\square$

Now we turn to the case of subobjects of direct sums. Let $\alpha, \beta$ be direct sums and let $\alpha^{\prime}, \beta^{\prime}$ denote subobjects of $\alpha, \beta$, correspondingly. Then we have

$$
\alpha^{\prime}(\cdot)=W^{*} \alpha(\cdot) W, \beta^{\prime}(\cdot)=V^{*} \beta(\cdot) V, W \in\left(\alpha^{\prime}, \alpha\right), V \in\left(\beta^{\prime}, \beta\right), W^{*} W=V^{*} V=1 .
$$

Now we put

$$
J\left(\alpha^{\prime}, \beta^{\prime}\right):=\phi(W \times V)^{*} J(\alpha, \beta) \phi(W) \otimes \phi(V) .
$$

Note that

$$
\alpha^{\prime} \beta^{\prime}(\cdot)=W^{*} \times V^{*}(\alpha \beta)(\cdot) W \times V
$$

Then we have

4.4.LEMMA. The isometries (11) satisfy the properties (C1)-(C4).

Proof. (C1) is obvious. (C3): Let $\alpha_{1}^{\prime}, \beta_{1}^{\prime}$ be subobjects of $\alpha_{1}, \beta_{1}$, respectively:

$$
\begin{gathered}
\alpha_{1}^{\prime}(\cdot)=W_{1}^{*} \alpha_{1}(\cdot) W_{1}, \quad \beta_{1}^{\prime}(\cdot)=V_{1}^{*} \beta_{1}(\cdot) V_{1}^{\prime}, \quad W_{1} \in\left(\alpha_{1}^{\prime}, \alpha_{1}\right), \quad V_{1} \in\left(\beta_{1}^{\prime}, \beta_{1}\right), \\
W_{1}^{*} W_{1}=V_{1}^{*} V_{1}=1 .
\end{gathered}
$$

Choose $A \in\left(\alpha^{\prime}, \alpha_{1}^{\prime}\right), B \in\left(\beta^{\prime}, \beta_{1}^{\prime}\right)$. Then

$$
W_{1} A W^{*} \in\left(\alpha, \alpha_{1}\right), V_{1} B V^{*} \in\left(\beta, \beta_{1}\right)
$$

and we have, according to Lemma 4.3.,

$$
J\left(\alpha_{1}, \beta_{1}\right) \phi\left(W_{1} A W^{*}\right) \otimes \phi\left(V_{1} B V^{*}\right)=\phi\left(W_{1} A W^{*} \times V_{1} B V^{*}\right) J(\alpha, \beta) .
$$


Multiplying this equation from the left by $\phi\left(W_{1}^{*} \times V_{1}^{*}\right)$, and from the right by $\phi(W \otimes V)$ we obtain

$$
\begin{gathered}
\phi\left(W_{1}^{*} \times V_{1}^{*}\right) J\left(\alpha_{1}, \beta_{1}\right) \phi\left(W_{1}\right) \otimes \phi\left(V_{1}\right) \cdot \phi(A) \otimes \phi(B)= \\
=\phi(A \times B) \phi\left(W^{*} \times V^{*}\right) J(\alpha, \beta) \phi(W) \otimes \phi(V)
\end{gathered}
$$

or

$$
J\left(\alpha_{1}^{\prime}, \beta_{1}^{\prime}\right) \phi(A) \otimes \phi(B)=\phi(A \times B) J(\alpha, \beta) .
$$

(C2): Let $\alpha^{\prime}, \beta^{\prime}$ be as before and let $\gamma^{\prime}$ be a subobject of $\gamma$, i.e. $\gamma^{\prime}(\cdot)=U^{*} \gamma(\cdot) U, U \in$ $\left(\gamma^{\prime}, \gamma\right), U^{*} U=1$. We calculate

$$
\begin{gathered}
J\left(\alpha^{\prime} \beta^{\prime}, \gamma^{\prime}\right)\left(J\left(\alpha^{\prime}, \beta^{\prime}\right) \otimes 1_{\mathcal{H}_{\gamma^{\prime}}}\right)= \\
\phi((W \times V) \times U)^{*} J(\alpha \beta, \gamma)(\phi(W \times V) \otimes \phi(U))\left(\phi(W \times V)^{*} J(\alpha, \beta)(\phi(W) \otimes \phi(V)) \otimes 1_{\mathcal{H}_{\gamma^{\prime}}}\right) \\
=\phi(W \times V \times U)^{*} J(\alpha \beta, \gamma)\left(\phi\left((W \times V)(W \times V)^{*}\right) J(\alpha, \beta)(\phi(W) \otimes \phi(V)) \otimes \phi(U)\right)= \\
=\phi(W \times V \times U)^{*} J(\alpha \beta, \gamma)\left(\phi\left(W W^{*} \times V V^{*}\right) J(\alpha, \beta)(\phi(W) \otimes \phi(V)) \otimes \phi(U)\right) \\
=\phi(W \times V \times U)^{*} J(\alpha \beta, \gamma)\left(J(\alpha, \beta) \otimes 1_{\mathcal{H}_{\gamma}}\right) \phi(W) \otimes \phi(V) \otimes \phi(U) \\
=\phi(W \times V \times U)^{*} J(\alpha, \beta \gamma)\left(1_{\mathcal{H}_{\alpha}} \otimes J(\beta, \gamma)\right) \phi(W) \otimes \phi(V) \otimes \phi(U)
\end{gathered}
$$

because of Lemma 4.3. and finally this equals $J\left(\alpha^{\prime}, \beta^{\prime} \gamma^{\prime}\right)\left(1_{\mathcal{H}_{\alpha^{\prime}}} \otimes J\left(\beta^{\prime}, \gamma^{\prime}\right)\right)$.

(C4): We have to prove

$$
J\left(\beta^{\prime}, \alpha^{\prime}\right) \Theta\left(\mathcal{H}_{\alpha^{\prime}}, \mathcal{H}_{\beta^{\prime}}\right)=\phi\left(\epsilon\left(\alpha^{\prime}, \beta^{\prime}\right)\right) J\left(\alpha^{\prime}, \beta^{\prime}\right) .
$$

So we calculate the left hand side, it equals

$$
\begin{gathered}
\phi(V \times W)^{*} J(\beta, \alpha) \phi(V) \otimes \phi(W) \Theta\left(\mathcal{H}_{\alpha^{\prime}}, \mathcal{H}_{\beta^{\prime}}\right)=\propto(V \times W)^{*} J(\beta, \alpha) \Theta\left(\mathcal{H}_{\alpha} . \mathcal{H}_{\beta}\right) \phi(W) \otimes \phi(V) \\
=\phi(V \times W)^{*} \phi(\epsilon(\alpha, \beta)) J(\alpha, \beta) \phi(W) \otimes \phi(V)= \\
=\phi\left(\epsilon\left(\alpha^{\prime}, \beta^{\prime}\right)\right) \phi\left(W^{*} \times V^{*}\right) J(\alpha, \beta) \phi(W) \otimes \phi(V)=\phi\left(\epsilon\left(\alpha^{\prime}, \beta^{\prime}\right)\right) J\left(\alpha^{\prime}, \beta^{\prime}\right) . \square
\end{gathered}
$$

The third step consists in the construction of a unique function

$$
\{\alpha, \beta\} \rightarrow J(\alpha, \beta), \quad \alpha, \beta \in \mathcal{T}
$$

such that $J(\alpha, \beta)$ satisfies all the properties (C1)-(C4). First let $\alpha, \beta$ be fixed. We consider the system of all isometries $\Phi$ from $\mathcal{H}_{\alpha} \otimes \mathcal{H}_{\beta}$ onto $\mathcal{H}_{\alpha \beta}$. It is denoted by $M(\alpha, \beta)$. Obviously, $M(\alpha, \beta)$ is a compact space (essentially the unitary group of all $d(\alpha) d(\beta) \times d(\alpha) d(\beta)$-matrices $)$. Then the cartesian product

$$
M:=\prod_{\alpha, \beta \in T} M(\alpha, \beta)
$$

is a compact space w.r.t the Tychonov topology.

Let $\mathcal{T}_{\text {fin }}:=\left\{\alpha_{1}, \alpha_{2}, \ldots, \alpha_{r}\right\}$ be a finite set of objects. Let $M\left(\mathcal{T}_{\text {fin }}\right) \subset M$ denote the subset consisting of all functions $J(\rho, \sigma)$ such that (C1)-(C4) is satisfied if $\rho, \sigma \in \mathcal{T}_{\text {fin }}$. The foregoing considerations (the first and the second step) show that $M\left(\mathcal{T}_{\text {fin }}\right) \neq \emptyset$ for each $\mathcal{T}_{\text {fin }}$, because the elements of $\mathcal{T}_{\text {fin }}$ are dominated by some special object $\rho$. 
Note that the intersection $\bigcap_{\mathcal{T}_{\text {fin }} \in \mathcal{C}} M\left(\mathcal{T}_{\text {fin }}\right)$ for a finite collection $\mathcal{C}=\left\{\mathcal{T}_{\text {fin }}\right\}$ is also nonempty because

$$
\bigcap_{\mathcal{T}_{\text {fin }} \in \mathcal{C}} M\left(\mathcal{T}_{f i n}\right) \supseteq M\left(\bigcup_{\mathcal{T}_{f i n} \in \mathcal{C}} \mathcal{T}_{f i n}\right)
$$

and $\bigcup_{\mathcal{T}_{\text {fin }} \in \mathcal{C}} \mathcal{T}_{\text {fin }}$ is again a finite subset of objects. Therefore we obtain

4.5.LEMMA. There is a function $J(\alpha, \beta)$ satisfying all the properties (C1)-(C4).

Proof. It is obvious because, according to Cantor's theorem,

$$
\bigcap_{\mathcal{T}_{f i n}} M\left(\mathcal{T}_{f i n}\right) \neq \emptyset
$$

where now $\mathcal{T}_{\text {fin }}$ runs through all finite subsets of abjects. A member $J(\cdot, \cdot) \in$ $\cap M\left(\mathcal{T}_{\text {fin }}\right)$ satisfies the properties $(\mathrm{C} 1)-(\mathrm{C} 4)$ for all $\alpha, \beta \in \mathcal{T}$.

We note that the argument using Cantor's theorem is already used by Doplicher/Roberts in the course of their treatment.

Now the goal of the second part of this section is the introduction of a product structure in $\mathcal{F}_{0}$, using the special isometries $J(\alpha, \beta): \mathcal{H}_{\alpha} \otimes \mathcal{H}_{\beta} \rightarrow \mathcal{H}_{\alpha \beta}$ constructed before.

Let $\Phi \in \mathcal{H}_{\rho}, \Psi \in \mathcal{H}_{\sigma}$, where $\rho, \sigma$ are irreducible. Then we define

$$
\Phi \cdot \Psi:=J(\rho, \sigma)(\Phi \otimes \Psi) .
$$

The extension of this definition to arbitrary elements of $\mathcal{F}_{0}$ is given by the distributive law. A straightforward calculation shows then that (12) is also true for elements of arbitrary Hilbert spaces $\mathcal{H}_{\alpha}, \mathcal{H}_{\beta}$ (see e.g. $[6$, p.365]).

4.6.PROPOSITION. The product in $\mathcal{F}_{0}$, defined by (12) and by its extension by the distributive law, is associative.

Proof. Obvious by the definition, because the tensor product is associative and (C2) holds.

We remark that corresponding formulas already appear in the treatment of Doplicher/Roberts: the formulas (SM1-SM3) and (6.2) in [2,p.303].

\section{The $*$-structure in the algebra $\mathcal{F}_{0}$}

The introduction of a $*$-structure in $\mathcal{F}_{0}$ consists of two steps. In the first one we introduce the concept of a conjugated basis in the Hilbert space $\mathcal{H}_{\bar{\rho}}$ where $\bar{\rho}$ denotes the representer of the conjugated class w.r.t. $\rho$.

Let $R_{\rho} \in(\iota, \bar{\rho} \rho)$ be a conjugate belonging to the pair $\rho, \bar{\rho}$. Then $R_{\rho} \in \mathcal{H}_{\bar{p} p}$ holds and therefore

$$
R_{p}=J(\bar{\rho}, \rho) u, \quad u \in \mathcal{H}_{\bar{\rho}} \otimes \mathcal{H}_{\rho} .
$$

We choose an orthonormal basis $\left\{\Phi_{k}\right\}_{k=1}^{d}$ in $\mathcal{H}_{\rho}$ and an orthonormal basis $\left\{\Psi_{j}\right\}_{j=1}^{d}$ in $\mathcal{H}_{\bar{\rho}}$, where $d=d(\rho)=d(\bar{\rho})$. Then

$$
u=\sum_{j, k} u_{j k} \Psi_{j} \otimes \Phi_{k}
$$


where $\left\{u_{j k}\right\}$ is a $d \times d$-matrix with complex entries. Now we have

5.1.LEMMA. The $d \times d$-matrix $\left\{u_{j k}\right\}$ is unitary.

Proof. We use the relation

$$
R_{\rho}^{*} \times 1_{\bar{\rho}} \cdot 1_{\bar{\rho}} \times S_{\rho}=1_{\bar{\rho}}
$$

where $S_{\rho}=\epsilon(\bar{\rho}, \rho) R_{\rho}$. We have $S_{\rho}=J(\rho, \bar{\rho}) v$ with $v \in \mathcal{H}_{\rho} \otimes \mathcal{H}_{\bar{\rho}}$ and

$$
J(\rho, \bar{\rho}) \Theta\left(\mathcal{H}_{\bar{\rho}}, \mathcal{H}_{\rho}\right) u=\phi(\epsilon(\bar{\rho}, \rho)) J(\bar{\rho}, \rho) u=\epsilon(\bar{\rho}, \rho) R_{\rho}=S_{\rho},
$$

therefore $v=\Theta\left(\mathcal{H}_{\bar{\rho}}, \mathcal{H}_{\rho}\right) u$. We have $\phi\left(1_{\bar{\rho}} \times S_{\rho}\right) \in \mathcal{L}\left(\mathcal{H}_{\bar{\rho}} \rightarrow \mathcal{H}_{\bar{\rho} \rho \bar{\rho}}\right)$ hence $\phi\left(1_{\bar{\rho}} \times S_{\rho}\right) x_{\bar{\rho}}=$ $1_{\bar{\rho}} \times S_{\rho} \cdot x_{\bar{\rho}}, x_{\bar{\rho}} \in \mathcal{H}_{\bar{\rho}}$. Furthermore,

$$
\phi\left(1_{\bar{\rho}} \times S_{\rho}\right) 1_{\bar{\rho}}=\phi\left(1_{\bar{\rho}} \times S_{p}\right) J(\bar{\rho}, \iota)=J(\bar{\rho}, \rho \bar{\rho}) \phi\left(1_{\bar{\rho}} \otimes \phi\left(S_{p}\right)\right.
$$

hence

$$
\begin{aligned}
& \phi\left(1_{\bar{\rho}} \times S_{\rho}\right) x_{\bar{\rho}}=J(\bar{\rho}, \rho \bar{\rho}) x_{\bar{\rho}} \otimes S_{\rho}=J(\bar{\rho}, \rho \bar{\rho})\left(x_{\bar{\rho}} \otimes J(\rho, \bar{\rho}) v\right) \\
= & J(\bar{\rho}, \rho \bar{\rho})\left(1_{\bar{\rho}} \otimes J(\rho, \bar{\rho})\right) x_{\bar{\rho}} \otimes v=J(\bar{\rho} \rho, \bar{\rho})\left(J(\bar{\rho}, \rho) \otimes 1_{\bar{\rho}}\right) x_{\bar{\rho}} \otimes v .
\end{aligned}
$$

Now there is a representation $v=\sum_{j, k} v_{j k} \Phi_{j} \otimes \Psi_{k}$ and replacing $v$ by this expression we obtain

$$
\begin{gathered}
\phi\left(1_{\bar{\rho}} \times S_{\rho}\right) x_{\bar{\rho}}=J(\bar{\rho} \rho, \bar{\rho})\left(J(\bar{\rho}, \rho) \otimes 1_{\bar{\rho}}\right) x_{\bar{\rho}} \otimes \sum_{j, k} v_{j k} \Phi_{j} \otimes \Psi_{k} \\
=J(\bar{\rho} \rho, \bar{\rho})\left(J(\bar{\rho}, \rho) \otimes 1_{\bar{\rho}}\right) \sum_{j, k} v_{j k} x_{\bar{\rho}} \otimes \Phi_{j} \otimes \Psi_{k} \\
=J(\bar{\rho} \rho, \bar{\rho}) \sum_{j, k} v_{j k}\left(J(\bar{\rho}, \rho) x_{\bar{\rho}} \otimes \Phi_{j}\right) \otimes \Psi_{k}
\end{gathered}
$$

and

$$
\begin{gathered}
\phi\left(R_{\rho}^{*} \times 1_{\bar{\rho}}\right) \phi\left(1_{\bar{\rho}} \times S_{\rho}\right) x_{\bar{\rho}}= \\
\phi\left(R_{\rho}^{*} \times 1_{\bar{\rho}}\right) J(\bar{\rho} \rho, \bar{\rho}) \sum_{j, k} v_{j k}\left(J(\bar{\rho}, \rho) x_{\bar{\rho}} \otimes \Phi_{j}\right) \otimes \Psi_{k} \\
=J(\iota, \bar{\rho}) \phi\left(R_{\rho}^{*}\right) \otimes \phi\left(1_{\bar{\rho}}\right) \sum_{j, k} v_{j k}\left(J(\bar{\rho}, \rho) x_{\bar{\rho}} \otimes \Phi_{j}\right) \otimes \Psi_{k} \\
=\phi\left(R_{\rho}^{*}\right) \otimes \phi\left(1_{\bar{\rho}}\right) \sum_{j, k} v_{j k}\left(J(\bar{\rho}, \rho) x_{\bar{\rho}} \otimes \Phi_{j}\right) \otimes \Psi_{k} .
\end{gathered}
$$

Now for $x \in \mathcal{H}_{\bar{\rho} \rho}, y \in \mathcal{H}_{\bar{\rho}}$ we have

$$
\phi\left(R_{\rho}^{*}\right) \otimes \phi\left(1_{\bar{\rho}}\right)(x \otimes y)=\phi\left(R_{\rho}^{*}\right) x \otimes \phi\left(1_{\bar{\rho}}\right) y=\left(R_{\rho}, x\right)_{\mathcal{H}_{\bar{\rho} \rho}} 1 \otimes y=\left(R_{\rho}, x\right)_{\mathcal{H}_{\bar{\rho} \rho}} y
$$

because $\phi\left(R_{\rho}^{*}\right) x=R_{\rho}^{*} \cdot x=\left(R_{\rho}, x\right)_{\mathcal{H}_{\bar{\rho} \rho}} 1$. In particular, we put $x:=J(\bar{\rho}, \rho)\left(x_{\bar{\rho}} \infty\right.$ $\left.\Phi_{j}\right), y:=\Psi_{k}$. Then we obtain

$$
\phi\left(R_{\rho}^{*}\right) \otimes \phi\left(1_{\bar{\rho}}\right)\left(J(\bar{\rho}, \rho)\left(x_{\bar{\rho}} \otimes \Phi_{j}\right)\right) \otimes \Psi_{k}=\left(R_{\rho}, J(\bar{\rho}, \rho)\left(x_{\bar{\rho}} \otimes \Phi_{j}\right)\right)_{\mathcal{H}_{\bar{\rho} \rho}} \Psi_{k}=
$$




$$
\begin{gathered}
=\left(J(\bar{\rho}, \rho) u, J(\bar{\rho}, \rho)\left(x_{\bar{\rho}} \otimes \phi_{j}\right)\right)_{\mathcal{H}_{\bar{\rho} \rho}} \Psi_{k}=\left(u, x_{\bar{\rho}} \otimes \Phi_{j}\right)_{\mathcal{H}_{\bar{\rho}} \otimes \mathcal{H}_{\rho}} \Psi_{k}= \\
=\left(\sum_{j^{\prime}, k^{\prime}} v_{j^{\prime} k^{\prime}} \Psi_{k^{\prime}} \otimes \Phi_{j^{\prime}}, x_{\bar{\rho}} \otimes \Phi_{j}\right)_{\mathcal{H}_{\bar{\rho}} \otimes \mathcal{H}_{\rho}} \Psi_{k}=\sum_{j^{\prime}, k^{\prime}} \bar{v}_{j^{\prime} k^{\prime}}\left(\Psi_{k^{\prime}}, x_{\bar{\rho}}\right)_{\mathcal{H}_{\bar{\rho}}}\left(\Phi_{j^{\prime}}, \Phi_{j}\right)_{\mathcal{H}_{\rho}} \Psi_{k}= \\
=\sum_{k^{\prime}} \bar{v}_{j k^{\prime}}\left(\Psi_{k^{\prime}}, x_{\bar{\rho}}\right)_{\mathcal{H}_{\bar{\rho}}}
\end{gathered}
$$

hence

$$
\begin{gathered}
\phi\left(R_{\rho}^{*} \times 1_{\bar{\rho}}\right) \phi\left(1_{\bar{\rho}} \times S_{\rho}\right) x_{\bar{\rho}}= \\
\sum_{j, k} v_{j k} \phi\left(R_{\rho}^{*}\right) \otimes \phi\left(1_{\bar{\rho}}\right)\left(J(\bar{\rho}, \rho) x_{\bar{\rho}} \otimes \Phi_{j}\right) \otimes \Psi_{k}=\sum_{j, k} v_{j k}\left(\sum_{k^{\prime}} \bar{v}_{j k^{\prime}}\left(\Psi_{k^{\prime}}, x_{\bar{\rho}}\right)_{\mathcal{H}_{\bar{\rho}}}\right) \Psi_{k}= \\
\sum_{k}\left(\sum_{j, k^{\prime}} v_{j k} \bar{v}_{j k^{\prime}}\left(\Psi_{k^{\prime}}, x_{\bar{\rho}}\right)_{\mathcal{H}_{\bar{\rho}}}\right) \Psi_{k}=x_{\bar{\rho}}=\sum_{k}\left(\Psi_{k}, x_{\bar{\rho}}\right)_{\mathcal{H}_{\bar{\rho}}} \Psi_{k} .
\end{gathered}
$$

This implies

$$
\sum_{j, k^{\prime}} v_{j k} \bar{v}_{j k^{\prime}}\left(\Psi_{k^{\prime}}, x_{\bar{\rho}}\right)_{\mathcal{H}_{\bar{\rho}}}=\left(\Psi_{k}, x_{\bar{\rho}}\right)_{\mathcal{H}_{\bar{\rho}}}, \quad x_{\bar{\rho}} \in \mathcal{H}_{\bar{\rho}}
$$

i.e.

$$
\sum_{j, k^{\prime}} v_{j k} \bar{v}_{j k^{\prime}} \Psi_{k^{\prime}}=\Psi_{k}, \quad k=1,2, \ldots, d
$$

or

$$
\sum_{j} v_{j k} \bar{v}_{j k^{\prime}}=\delta_{k k^{\prime}}
$$

i.e. the matrix $\left\{v_{j k}\right\}$ is unitary, hence $\left\{u_{j k}\right\}$ is also unitary. $\square$

Using the second conjugation relation

$$
S_{\rho}^{*} \times 1_{\rho} \cdot 1_{\rho} \times R_{\rho}=1_{\rho},
$$

one obtains the same result, i.e. the unitarity of the matrix $\left\{u_{j k}\right\}$ of the vector $u \in \mathcal{H}_{\bar{p}} \otimes \mathcal{H}_{p}$ w.r.t. arbitrary chosen orthonormal bases. However, in this case the unitarity relation reads

$$
\sum_{k} v_{j k} \bar{v}_{j^{\prime} k}=\delta_{j j^{\prime}}
$$

i.e. the difference is only that between the orthogonality of rows and columns.

Using the unitary matrix $\left\{u_{j k}\right\}$ one can define a transformed orthonormal basis in $\mathcal{H}_{\widetilde{\rho}}$ by the formula

$$
\Psi_{j}^{\prime}:=\sum_{j^{\prime}} u_{j^{\prime} j} \Psi_{j^{\prime}}
$$

Obviously, $\left\{\Psi_{j}^{\prime}\right\}_{j=1}^{d}$ is an orthonormal basis in $\mathcal{H}_{\bar{\rho}}$. However, w.r.t. this basis the formula for the vector $u \in \mathcal{H}_{\vec{\rho}} \otimes \mathcal{H}_{\rho}$ reads now

$$
u=\sum_{j} \Psi_{j}^{\prime} \otimes \Phi_{j}
$$


We define the concept conjugated basis by this relation.

5.2.DEFINITION. Let $\left\{\Phi_{j}\right\}_{j=1}^{d}$ be an orthonormal basis of the Hilbert space $\mathcal{H}_{\rho}$. An orthonormal basis $\left\{\Psi_{k}\right\}_{k=1}^{d}$ of $\mathcal{H}_{\bar{p}}$ is said to be conjugated w.r.t. $\left\{\Phi_{j}\right\}_{j=1}^{d}$ if

$$
u=\sum_{j=1}^{d} \Psi_{j} \otimes \Phi_{j}, \quad R_{\rho}=J(\bar{\rho}, \rho) u .
$$

Then the foregoing considerations based on Lemma 5.1. can be formulated as follows:

5.3.LEMMA. Let $\rho \in \mathcal{T}$ and let $\bar{\rho} \in \mathcal{T}$ be conjugated w.r.t. $\rho$. Let $\left\{\Phi_{\rho j}\right\}_{j=1}^{d(\rho)}$ be an orthonormal basis in $\mathcal{H}_{\rho}$. Then there exists a conjugated basis $\left\{\Psi_{\bar{\rho} j}\right\}_{j=1}^{d(\rho)}$ in $\mathcal{H}_{\bar{\rho}}$, i.e.

$$
R_{\rho}=J(\bar{\rho}, \rho)\left(\sum_{j=1}^{d(\rho)} \Psi_{\bar{\rho} j} \otimes \Phi_{\rho j}\right) .
$$

In general a conjugated basis is not unique. However, note that if $\rho$ is irreducible then $R_{\rho}$ hence $\mathrm{u}$, where $R_{\rho}=J(\bar{\rho}, \rho) u$, are unique up to a phase factor $\lambda,|\lambda|=1$. Therefore we obtain

5.4.LEMMA. If $\rho$ is irreducible then the conjugated basis is unique up to a phase factor $\lambda,|\lambda|=1$.

Proof. Let $\rho$ be irreducible. Then $R_{\rho}$ is unique up to a factor $\lambda,|\lambda|=1$ (see Doplicher/Roberts [2,Lemma 2.2.,p.165]). Then $u$ is also unique up to this factor. Let $\Phi_{j}$ be an orthonormal basis of $\mathcal{H}_{\rho}$ and let $\Psi_{k}, \Psi_{k}^{\prime}$ be conjugated bases. Then we have $\sum_{j} \Psi_{j}^{\prime} \otimes \Phi_{j}=\lambda \sum_{j} \Psi j \otimes \Phi_{j},|\lambda|=1$ hence $\sum_{j}\left(\Psi_{j}^{\prime}-\lambda \Psi_{j}\right) \otimes \Phi_{j}=0$ and it follows that $\Psi_{j}^{\prime}=\lambda \Psi_{j}$.

Now we proceed to the second step, the definition of the *-structure in $\mathcal{F}_{0}$.

5.5.DEFINITION. Let $\rho \in \hat{\mathcal{T}}$ be irreducible and let $\Phi_{\rho j}, j=1, \ldots, d$ be an orthonormal basis in $\mathcal{H}_{\rho}$. The conjugated basis in $\mathcal{H}_{\bar{\rho}}$ is denoted by $\Phi_{\bar{\rho} j}, j=1, \ldots, d$. Then $\Phi_{\rho j}^{*}$ is defined by

$$
\Phi_{\rho j}^{*}:=R_{\rho}^{*} \Phi_{\bar{p} j}, \quad j=1, \ldots, d .
$$

Note that this definition makes sense. Namely, if $\Psi_{\bar{\rho} j}$ is a second conjugated basis then $\Psi_{\bar{\rho} j}=\lambda \Phi_{\bar{\rho} j},|\lambda|=1$, and $\sum_{j} \Psi_{\bar{\rho} j} \otimes \Phi_{\rho j}=\lambda \sum_{j} \Phi_{\bar{\rho} j} \otimes \Phi_{\rho j}$ hence $J(\bar{\rho}, \rho) \sum_{j} \Psi_{\bar{\rho} j} \otimes$ $\Phi_{\rho j}=\lambda J(\bar{\rho}, \rho) \sum_{j} \Phi_{\bar{\rho} j} \otimes \Phi_{\rho j}$ or $\tilde{R}_{\rho}=\lambda R_{\rho}$ and $\tilde{R}_{\rho}^{*} \Psi_{\bar{\rho} j}=\bar{\lambda} R_{\rho}^{*} \lambda \Phi_{\bar{\rho} j}=|\lambda|^{2} R_{\rho}^{*} \Phi_{\bar{\rho} j}=$ $R_{\rho}^{*} \Phi_{\bar{\rho} j}$.

Additionally we define

$$
\left(A \Phi_{\rho j}\right)^{*}:=\Phi_{\rho j}^{*} A^{*}
$$

and extend the definition by linearity to the whole algebra $\mathcal{F}_{0}$. We have

5.6.LEMMA. The relations

$$
\begin{aligned}
\left(\Phi_{\rho j}^{*}\right)^{*} & =\Phi_{\rho j}, \\
\left(\Phi_{\rho j} \cdot \Phi_{\rho^{\prime} j^{\prime}}\right)^{*} & =\Phi_{\rho^{\prime} j^{\prime}}^{*} \cdot \Phi_{\rho j}^{*},
\end{aligned}
$$


hold.

Proof. First we prove (13): We have

$$
\begin{gathered}
\left(\Phi_{\rho j}^{*}\right)^{*}=\left(R_{\rho}^{*} \Phi_{\bar{\rho} j}\right)^{*}=\Phi_{\bar{\rho} j}^{*} R_{\rho}=R_{\bar{\rho}}^{*} \Phi_{\rho j} R_{\rho}= \\
R_{\bar{\rho}}^{*} \rho\left(R_{\rho}\right) \Phi_{\rho j}=R_{\bar{\rho}}^{*} \times 1_{\rho} \cdot 1_{\rho} \times R_{\rho} \cdot \Phi_{\rho j}=\Phi_{\rho j}
\end{gathered}
$$

because of the conjugation relation (T8) for $R_{\rho}$, where $R_{\bar{\rho}}:=S_{\rho}$.

Second we prove (14): Here we have

$$
\Phi_{\rho j} \cdot \Phi_{\rho^{\prime} j^{\prime}}=\sum_{k} W_{k} \psi_{k}, \quad \psi_{k}:=\sum_{j} \lambda_{j} \Phi_{\tau_{k} j} \in \mathcal{H}_{\tau_{k}}, \quad W_{k} \in\left(\tau_{k}, \rho \rho^{\prime}\right)
$$

where the $\tau_{k} \in \hat{\mathcal{T}}$ are irreducible. Therefore

$$
\left(\Phi_{\rho j} \cdot \Phi_{\rho^{\prime} j^{\prime}}\right)^{*}=\sum_{k} \psi_{k}^{*} W_{k}^{*}=\sum_{k} R_{\tau_{k}}^{*} \bar{\psi}_{k} W_{k}^{*}=\sum_{k} R_{\tau_{k}}^{*} \bar{\tau}_{k}\left(W_{k}^{*}\right) \bar{\psi}_{k},
$$

where $\bar{\psi}_{k}:=\sum_{j} \bar{\lambda}_{j} \Phi_{\bar{\tau}_{k} j}$. On the other hand we get

$$
\Phi_{\rho^{\prime} j^{\prime}}^{*} \Phi_{\rho j}^{*}=R_{\rho^{\prime}}^{*} \Phi_{\overline{\rho^{\prime} j^{\prime}}} R_{\rho}^{*} \Phi_{\bar{\rho} j}=R_{\rho^{\prime}}^{*} \overline{\rho^{\prime}}\left(R_{\rho}^{*}\right) \Phi_{\overline{\rho^{\prime} j^{\prime}}} \Phi_{\overline{\rho^{\prime} j}}
$$

and we have

$$
\Phi_{\bar{\rho}^{\prime} j^{\prime}} \Phi_{\bar{\rho} j}=\sum_{k} \dot{W}_{k} \bar{\psi}_{k}
$$

where $\tilde{W}_{k}$ is the conjugated arrow w.r.t. $W_{k}, \tilde{W}_{k} \in\left(\overline{\tau_{k}}, \overline{\rho^{\prime}} \bar{\rho}\right)$, see $[2$, p.167] or $[6, p .257]$. So we obtain

$$
\Phi_{\rho^{\prime} j^{\prime}}^{*} \Phi_{\rho j}^{*}=\sum_{k} R_{\rho^{\prime}}^{*}{\overline{\rho^{\prime}}}\left(R_{\rho}^{*}\right) \hat{W}_{k} \bar{\psi}_{k}
$$

Comparing (15) and (16) we see that it is sufficient to prove

$$
R_{\tau}^{*} \bar{\tau}\left(W^{*}\right)=R_{\rho^{\prime}}^{*} \overline{\rho^{\prime}}\left(R_{p}^{*}\right) \grave{W}
$$

where $\tau$ is irreducible, $W \in\left(\tau, \rho \rho^{\prime}\right)$, and $\tilde{W} \in\left(\bar{\tau}, \overline{\rho^{\prime}} \bar{\rho}\right)$ is the conjugated arrow. Note that

$$
R_{\rho^{\prime}}^{*} \overline{\rho^{\prime}}\left(R_{\rho}^{*}\right)=R_{\rho \rho^{\prime}}^{*}
$$

This means that it is sufficient to prove

$$
R_{\tau}^{*} \bar{\tau}\left(W^{*}\right)=R_{\sigma}^{*} \tilde{W}
$$

where $\tau$ is irreducible, $\sigma$ arbitrary, $W \in(\tau, \sigma), \tilde{W} \in(\bar{\tau}, \bar{\sigma})$ the conjugated arrow. But (17) is the characteristic relation for the conjugated arrow (see Doplicher/Roberts [2,Lemma 2.5.,p.167] or $[7,(12)$, p.257]).

The relations (13) and (14) ensure that the *-structure given by Definition 5.5 satisfies the usual properties, i.e. $\mathcal{F}_{0}$ is a $*$-algebra.

The orthonormal bases of the Hilbert spaces $\mathcal{H}_{\alpha}, \alpha \in \mathcal{T}$, satisfy important relations. 
5.7.PROPOSITION. Let $\alpha \in \mathcal{T}$ be arbitrary and let $\left\{\Phi_{\alpha j}\right\}_{j=1}^{d(\alpha)}$ be an orthonormal basis of $\mathcal{H}_{\alpha}$. Then the relations

$$
\begin{gathered}
\Phi_{\alpha j}^{*} \Phi_{\alpha k}=\delta_{j k} 1, \\
\sum_{j} \Phi_{\alpha j} \Phi_{\alpha j}^{*}=1
\end{gathered}
$$

hold.

Proof. First we prove (18) for $\rho \in \hat{\mathcal{T}}$. We have $\Phi_{\rho j}^{*} \Phi_{\rho k}=R_{\rho}^{*} \Phi_{\bar{\rho} j} \Phi_{\rho k}$. Note that

$$
\phi\left(R_{\rho}^{*}\right) X=R_{\rho}^{*} X=\left(R_{\rho}, X\right)_{\mathcal{H}_{\bar{\rho} \rho}} 1, \quad X \in \mathcal{H}_{\bar{\rho} \rho} .
$$

Thus

$$
\begin{gathered}
\Phi_{\rho j}^{*} \Phi_{\rho k}=\left(R_{\rho}, \Phi_{\bar{\rho} j} \Phi_{\rho k}\right)_{\mathcal{H}_{\bar{\rho} \rho}} 1= \\
=\left(\sum_{l=1}^{d(\rho)} \Phi_{\bar{\rho} l} \Phi_{\rho l}, \Phi_{\bar{\rho} j} \Phi_{\rho k}\right)_{\mathcal{H}_{\bar{\rho} \rho}} 1= \\
\sum_{l=1}^{d(\rho)}\left(\Phi_{\bar{\rho} l}, \Phi_{\bar{\rho} j}\right)_{\mathcal{H}_{\bar{\rho}}}\left(\Phi_{\rho l}, \Phi_{\rho k}\right)_{\mathcal{H}_{\rho}} 1=\sum_{l=1}^{d(\rho)} \delta_{l j} \delta_{l k} 1=\delta_{j k} 1 .
\end{gathered}
$$

Now, using the definition of the Hilbert spaces $\mathcal{H}_{\alpha}, \alpha \in \mathcal{T}$, it is easy to extend the formula to arbitrary $\alpha \in \mathcal{T}$.

Second we prove $(19)$, again first for $\rho \in \hat{\mathcal{T}}$. We calculate

$$
\begin{gathered}
\sum_{j=1}^{d(\rho)} \Phi_{\rho j} \Phi_{\rho j}^{*}=\sum_{j=1}^{d(\rho)} \Phi_{\rho j} R_{\rho}^{*} \Phi_{\bar{\rho} j}=\sum_{j=1}^{d(\rho)} \rho\left(R_{\rho}^{*}\right) \Phi_{\rho j} \Phi_{\bar{\rho} j} \\
=\rho\left(R_{\rho}^{*}\right) \sum_{j=1}^{d(\rho)} \Phi_{\rho j} \Phi_{\bar{\rho} j}=\rho\left(R_{\rho}^{*}\right) R_{\bar{\rho}}=1_{\rho} \times R_{\rho}^{*} \cdot R_{\bar{\rho}} \times 1_{\rho}=1_{\rho},
\end{gathered}
$$

according to the conjugation relation. Again, using the definition of the Hilbert spaces $\mathcal{H}_{\alpha}$, the formula can be easily extended to arbitrary $\alpha \in \mathcal{T}$.

Proposition 5.7. means that the Hilbert spaces $\mathcal{H}_{\alpha} \subset \mathcal{F}_{0}, \alpha \in \mathcal{T}$. are algebraic Hilbert spaces of the $*$-algebra $\mathcal{F}_{0}$. In particular, (19) means that the so-called support of $\mathcal{H}_{\alpha}$ equals 1 . Note that the Hilbert spaces $\mathcal{H}_{\rho}$ for irreducibles $\rho \in \hat{\mathcal{T}}$ together with $\mathcal{A}$ are generating for $\mathcal{F}_{0}$ w.r.t. $\mathcal{F}_{0}$ as an $\mathcal{A}$-leftmodule.

Recall that according to Propositions 3.1. and 3.2. we have bijections

$$
\begin{gathered}
\mathrm{Ob} \mathcal{I} \in \alpha \longleftrightarrow \mathcal{H}_{\alpha} \subset \mathcal{F}_{0}, \\
\mathcal{A} \supset(\alpha, \beta) \longleftrightarrow \phi((\alpha, \beta)) \subset \mathcal{L}\left(\mathcal{H}_{\alpha} \rightarrow \mathcal{H}_{\beta}\right) .
\end{gathered}
$$

Taking into account the results in sections 4 and 5 we can conclude that in fact by these bijections there is established a categorial isomorphism: First we have

$$
\mathcal{H}_{\alpha \beta}=\mathcal{H}_{\alpha} \cdot \mathcal{H}_{\beta}
$$


where $\mathcal{H}_{\alpha} \cdot \mathcal{H}_{\beta}$ denotes the algebraic Hilbert space spa $\left\{\mathcal{H}_{\alpha} \cdot \mathcal{H}_{\beta}\right\}$. Second, according to the original definition of the algebraic Hilbert spaces $\mathcal{H}_{\alpha}$, we get: If

$$
\gamma(\cdot)=V \alpha(\cdot) V^{*}+W \beta(\cdot) W^{*},
$$

where $V \in(\alpha, \gamma), W \in(\beta, \gamma), V V^{*}+W W^{*}=1, V^{*} V=W^{*} W=1$, then

$$
\mathcal{H}_{\gamma}=V \mathcal{H}_{\alpha}+W \mathcal{H}_{\beta}
$$

Third we obtain: the category $\left\{\mathcal{H}_{\alpha}\right\}_{\alpha}$ is equipped with a conjugation, given by the conjugation $\alpha \rightarrow \bar{\alpha}$.

Therefore, at this stage we can apply the Tannaka-Krein result. This means there is a compact group $\mathcal{K}$ such that $\hat{\mathcal{K}}$ is in one-to-one correspondence to $\left\{\mathcal{H}_{\rho}\right\}_{\rho}$ for a full collection of representers $\rho \in \hat{\mathcal{T}}$. So to each $\alpha \in \mathcal{T}$ we get a unitary representation $U_{\alpha}(\mathcal{K})$ of $\mathcal{K}$ on $\mathcal{H}_{\alpha}$ which is irreducible for $\alpha \in \hat{\mathcal{T}}$. Note that $\mathcal{K}$ acts on $\mathcal{H}_{\bar{\alpha}}, \alpha \in \mathcal{T}$, by the conjugated representation w.r.t. $\mathcal{H}_{\alpha}$, i.e.

$$
U_{\widetilde{\alpha}}(k)=\overline{U_{\alpha}(k)}, \quad k \in \mathcal{K},
$$

where - means complex conjugation of the representing matrices.

The special structure of $\mathcal{F}_{0}$ allows to define an action of $\mathcal{X}$ on $\mathcal{F}_{0}$ by the definition

$$
U(k)\left(\sum_{\rho, j} A_{\rho j} \Phi_{\rho j}\right):=\sum_{\rho, j} A_{\rho j} U_{\rho}(k) \Phi_{\rho j} .
$$

Obviously we have

$$
U(k)(X)=U_{\alpha}(k) X, \quad X \in \mathcal{H}_{\alpha},
$$

because from

$$
X=\sum_{j=1}^{r} B_{j} \Phi_{j}, B_{j} \in\left(\rho_{j}, \alpha\right), \Phi_{j} \in \mathcal{H}_{\rho_{j}}, \rho_{j} \text { irreducible }
$$

we obtain

$$
U(k)(X)=\sum_{j=1}^{\tau} B_{j} U_{\rho_{j}} \Phi_{j}=U_{\alpha}(k) \sum_{j=1}^{r} B_{j} \Phi_{j}=U_{\alpha}(k) X .
$$

Therefore it turns out that $U(k)$ is even an automorphism of $\mathcal{F}_{\mathbf{0}}$, because for $\Phi_{1} \in$ $\mathcal{H}_{\alpha}, \Phi_{2} \in \mathcal{H}_{\beta}$ we obtain

$$
U(k)\left(\Phi_{1} \cdot \Phi_{2}\right)=U_{\alpha \beta}(k)\left(\Phi_{1} \Phi_{2}\right)=\left(U_{\alpha}(k) \Phi_{1}\right)\left(U_{\beta}(k) \Phi_{2}\right)=U(k) \Phi_{1} \cdot U(k) \Phi_{2} .
$$

Moreover we have

$$
(U(k)(F))^{*}=U(k)\left(F^{*}\right), \quad F \in \mathcal{F}_{0} .
$$

Namely, let $\left\{\Phi_{\rho j}\right\}_{j=1}^{d(\rho)}$ be an orthonormal basis of $\mathcal{H}_{\rho}$ and

$$
U_{\rho}(k) \Phi_{\rho j}=\sum_{j^{\prime}=1}^{d(\rho)} u_{j^{\prime} j}^{(\rho)}(k) \Phi_{\rho j^{\prime}}
$$


Then we get

$$
\begin{gathered}
\left(U(k)\left(\Phi_{\rho j}\right)\right)^{*}=\left(U_{\rho}(k) \Phi_{\rho j}\right)^{*}=\left(\sum_{j^{\prime}=1}^{d(\rho)} u_{j^{\prime} j}^{(\rho)}(k) \Phi_{\rho j^{\prime}}\right)^{*} \\
=\sum_{j^{\prime}=1}^{d(\rho)} \overline{u_{j^{\prime} j}^{(\rho)}(k)} \Phi_{\rho j^{\prime}}^{*}=\sum_{j^{\prime}=1}^{d(\rho)} \overline{u_{j^{\prime} j}^{(\rho)}} R_{\rho}^{*} \Phi_{\bar{\rho} j^{\prime}}= \\
R_{\rho}^{*} \sum_{j^{\prime}=1}^{d(\rho)} \overline{u_{j^{\prime} j}^{(\rho)}} \Phi_{\bar{\rho} j^{\prime}}=R_{\rho}^{*}\left(U_{\bar{\rho}}(k) \Phi_{\bar{\rho} j}\right)= \\
R_{\rho}^{*} U(k)\left(\Phi_{\bar{\rho} j}\right)=U(k)\left(R_{\rho}^{*} \Phi_{\bar{\rho} j}\right)=U(k)\left(\Phi_{\rho j}^{*}\right) .
\end{gathered}
$$

This relation can be easily extended to be valid for arbitrary $F=\sum_{\rho, j} A_{\rho j} \Phi_{\rho j}$.

Furthermore, the fixed point algebra of $U(\mathcal{K})$ within $\mathcal{F}_{0}$ equals $\mathcal{A}$, because if $\mathcal{F}_{0} \ni F=\sum_{\rho, j} A_{\rho j} \Phi_{\rho j}$ and $U(k)(F)=F$ for all $k \in \mathcal{K}$ then we obtain

$$
\sum_{\rho, j} A_{\rho j} U_{\rho}(k) \Phi_{\rho j}=\sum_{\rho, j, j^{\prime}} A_{\rho j} u_{j^{\prime} j}^{(\rho)}(k) \Phi_{\rho j^{\prime}}=\sum_{\rho, j} A_{\rho j} \Phi_{\rho j}
$$

hence

$$
\sum_{j^{\prime}=1}^{d(\rho)} u_{j j^{\prime}}^{(\rho)}(k) A_{\rho . j^{\prime}}=A_{\rho j}
$$

follows for all $k \in \mathcal{K}, \rho \in \hat{\mathcal{T}}, j=1,2 \ldots, d(\rho)$. If $\rho \neq \iota$, by integration over $\mathcal{K}$ we get $A_{\rho j}=0$.

In the next section we characterize the group $\mathcal{K}$ as the group of all automorphisms of $\mathcal{F}_{0}$ leaving a characteristic $\mathcal{A}$-scalar product invariant.

\section{The $\mathcal{A}$-scalar product and the symmetry group}

Let $F, G \in \mathcal{F}_{0}$. Then $F=\sum_{\rho, j} A_{\rho j} \Phi_{\rho j}, G=\sum_{\rho, j} B_{\rho j} \Phi_{\rho j}$ where $\left\{\Phi_{\rho j}\right\}_{j=1}^{d(\rho)}$ denotes, as before, an orthonormal basis in $\mathcal{H}_{\rho} ; A_{\rho j}, B_{\rho j} \in \mathcal{A}$. Recall $\Phi_{\iota}=1$. The coefficient $A_{\iota}$ of $F$ is called the $\mathcal{A}$-component of $F$, resp. $B$, is the $\mathcal{A}$-component of $G$. We define an $\mathcal{A}$-scalar product in the $*$-algebra $F_{0}$ by

$$
\langle F, G\rangle_{\mathcal{A}}:=\sum_{\rho, j} \frac{1}{d(\rho)} A_{\rho j} B_{\rho j}^{*} .
$$

Obviously, $\langle F, G\rangle_{\mathcal{A}} \in \mathcal{A}$ and $\langle F, G\rangle_{\mathcal{A}}$ is independent of the special choice of the orthonormal bases in the Hilbert spaces $\mathcal{H}_{\rho}$. The $\mathcal{A}$-scalar product $\langle F, G\rangle_{\mathcal{A}}$ satisfies the properties

$$
\begin{gathered}
\langle A F, B G\rangle_{\mathcal{A}}=A\langle F, G\rangle_{\mathcal{A}} B^{*}, \\
\langle F, F\rangle_{\mathcal{A}} \geq 0, \\
\langle F, F\rangle_{\mathcal{A}}=0 \quad \text { iff } \quad F=0 .
\end{gathered}
$$

We have 
6.1.LEMMA. Let $F, G \in \mathcal{F}_{0}$. Then $\langle F, G\rangle_{\mathcal{A}}$ coincides with the $\mathcal{A}$-component of
$G^{*}$.

Proof. It is sufficient to prove this for $F:=\Phi_{\alpha j}, G:=\Phi_{\beta k}, \alpha, \beta \in \hat{\mathcal{T}}$ irreducible. Recall that there is a decomposition

$$
\Phi_{\alpha j} \cdot \Phi_{\beta k}=\sum_{r, l} K_{\alpha j \beta k}^{\tau l} \Phi_{\tau l}
$$

of the product $\Phi_{\alpha j} \cdot \Phi_{\beta k}$ where $K_{\alpha j \beta k}^{\tau l} \in(\tau, \alpha \beta)$. We have

$$
\Phi_{\alpha j} \Phi_{\beta k}^{*}=\Phi_{\alpha j} R_{\beta}^{*} \Phi_{\bar{\beta} k}=\alpha\left(R_{\beta}^{*}\right) \Phi_{\alpha, j} \Phi_{\bar{\beta} k} .
$$
Therefore, the $\mathcal{A}$-component of $\Phi_{\alpha j} \Phi_{\beta k}^{*}$ coincides with $\alpha\left(R_{\beta}^{*}\right) K_{\alpha j \bar{\beta} k}^{\iota}$. On the other
hand, by definition we have

$$
\left\langle\Phi_{\alpha j}, \Phi_{\beta k}\right\rangle_{\mathcal{A}}=\frac{1}{d(\alpha)} \delta_{\alpha \beta} \delta_{j k}
$$

So it is sufficient to prove

$$
\alpha\left(R_{\beta}^{*}\right) K_{\alpha j \bar{\beta} k}^{\iota}=\frac{1}{d(\alpha)} \delta_{\alpha \beta} \delta_{j k}
$$

We check easily that

$$
\alpha\left(R_{\beta}^{*}\right) K_{\alpha j \bar{\beta} k}^{\iota} \in(\beta, \alpha)
$$

because $K_{\alpha j \bar{\beta} k}^{\prime} \in(\beta, \alpha \bar{\beta} \beta)$ and $\alpha\left(R_{\beta}^{*}\right) \in(\alpha \bar{\beta} \beta, \alpha)$. Therefore we have: if $\alpha \neq \beta$ then $\alpha\left(R_{\beta}^{*}\right) K_{\alpha j \bar{\beta} k}^{\iota}=0$ and $(20)$ is true. Now let $\alpha=\beta$. Then we have to prove

$$
a\left(R_{\alpha}^{\star}\right) K_{\alpha j \bar{\alpha} k}=\frac{1}{d(\alpha)} \delta_{j k} 1
$$

Since the left hand side is a member of $(\alpha, \alpha)$, we have

$$
\alpha\left(R_{\alpha}^{*}\right) K_{\alpha j \bar{\alpha} k}^{\prime \iota}=\lambda_{j k} 1, \quad \lambda_{j k} \in \mathbf{C} .
$$

Note that

$$
K_{\alpha j \bar{\alpha} k}^{\iota}=J(\alpha, \bar{\alpha}) u_{j k}, \quad u_{j k} \in \mathcal{H}_{\alpha} \otimes \mathcal{H}_{\bar{\alpha}}, \quad u_{j k}=\sum_{p, q} c_{j k}^{p q} \Phi_{\alpha p} \otimes \Phi_{\bar{\alpha} q} .
$$

Let $X \in \mathcal{H}_{\alpha}$. We calculate

$$
\begin{gathered}
\alpha\left(R_{\alpha}^{*}\right) K_{\alpha j \bar{\alpha} k}^{\prime} X=1_{\alpha} \times R_{\alpha}^{*} \cdot J(\alpha \bar{\alpha}, \alpha)\left(K_{\alpha j \bar{\alpha} k}^{\prime \iota} \otimes X\right) \\
=1_{\alpha} \times R_{\alpha}^{*} \cdot J(\alpha \bar{\alpha}, \alpha)\left(J(\alpha, \bar{\alpha}) u_{j k} \otimes 1_{\mathcal{H}_{\alpha}} X\right) \\
=1_{\alpha} \times R_{\alpha}^{*} \cdot J(\alpha \bar{\alpha}, \alpha)\left(J(\alpha, \bar{\alpha}) \otimes 1_{\mathcal{H}_{\alpha}}\right)\left(u_{j k} \otimes X\right)= \\
1_{\alpha} \times R_{\alpha}^{*} \cdot J(\alpha, \bar{\alpha} \alpha)\left(1_{\mathcal{H}_{\alpha}} \otimes J(\bar{\alpha}, \alpha)\right)\left(\sum_{p, q} c_{j k}^{p q} \Phi_{\alpha p} \otimes \Phi_{\bar{\alpha} q} \otimes X\right)=
\end{gathered}
$$




$$
\begin{aligned}
& J(\alpha, \ell)\left(1_{\mathcal{H}_{\alpha}} \otimes R_{\alpha}^{*}\right)\left(1_{\mathcal{H}_{\alpha}} \otimes J(\bar{\alpha}, \alpha)\right)\left(\sum_{p, q} c_{j k}^{p q} \Phi_{\alpha p} \otimes \Phi_{\bar{\alpha} q} \otimes X\right)= \\
& \left(1_{\mathcal{H}_{\alpha}} \otimes R_{\alpha}^{*} J(\bar{\alpha}, \alpha)\right)\left(\sum_{p, q} c_{j k}^{p q} \Phi_{\alpha p} \otimes \Phi_{\bar{\alpha} q} \otimes X\right)=\sum_{p, q} c_{j k}^{p q} \Phi_{\alpha p}\left(R_{\alpha}, J(\bar{\alpha}, \alpha) \Phi_{\bar{\alpha}_{q}} \otimes X\right)_{\mathcal{H}_{\bar{\alpha} \alpha}}= \\
& \sum_{p, q} c_{j k}^{p q} \Phi_{\alpha p}\left(J(\bar{\alpha}, \alpha) \sum_{l=1}^{d(\alpha)} \Phi_{\bar{\alpha} l} \otimes \Phi_{\alpha l}, J(\bar{\alpha}, \alpha) \Phi_{\bar{\alpha} q} \otimes X\right)_{\mathcal{H}_{\bar{\alpha} \alpha}}= \\
& \sum_{p, q} c_{j k}^{p q} \Phi_{\alpha p} \sum_{l=1}^{d(\alpha)}\left(\Phi_{\bar{\alpha} l} \otimes \Phi_{\alpha l}, \Phi_{\bar{\alpha} q} \otimes X\right)_{\mathcal{H}_{\bar{\alpha}} \otimes \mathcal{H}_{\alpha}}=\sum_{p, q} c_{j k}^{p q} \Phi_{\alpha p} \sum_{l=1}^{d(\alpha)}\left(\Phi_{\bar{\alpha} l}, \Phi_{\bar{\alpha} q}\right)_{\mathcal{H}_{\bar{\alpha}}}\left(\Phi_{\alpha l}, X\right)_{\mathcal{H}_{\alpha}} \\
& =\sum_{p, q} c_{j k}^{p q} \Phi_{\alpha p}\left(\Phi_{\alpha q}, X\right)_{\mathcal{H}_{\alpha}}=\lambda_{j k} X .
\end{aligned}
$$

For $X:=\Phi_{\alpha s}$ we obtain the equation

$$
\sum_{p} c_{j k}^{p s} \Phi_{\alpha p}=\lambda_{j k} \Phi_{c r s}
$$

hence

follows. That is, we obtain

$$
c_{j k}^{p s}=\delta^{p s} \lambda_{j k}
$$

$$
u_{j k}=\lambda_{j k} \sum_{p} \Phi_{\alpha p} \otimes \Phi_{\bar{\alpha} p}
$$

and

$$
K_{\alpha, j \bar{\alpha} k}^{r \ell}=\lambda_{j k} J(\alpha, \bar{\alpha})\left(\sum_{p} \Phi_{\alpha p} \otimes \Phi_{\bar{\alpha} p}\right)=\lambda_{j k} R_{\bar{\alpha}} .
$$

Therefore we can write

$$
\Phi_{\alpha j} \cdot \Phi_{\bar{\alpha} k}=\lambda_{j k} R_{\bar{\alpha}}+\sum_{\gamma \neq \iota} \sum_{l} K_{\alpha j \bar{\alpha} k}^{\gamma l} \Phi_{\gamma l}
$$

In other words, we have: The $\mathcal{A}$-component of $\Phi_{\alpha j} \cdot \Phi_{\bar{\alpha} k}$ equals $\lambda_{j k} R_{\bar{\alpha}}$. From (21) we obtain

$$
R_{\bar{\alpha}}^{*} \cdot \Phi_{\alpha j} \cdot \Phi_{\bar{\alpha} k}=\lambda_{j k} R_{\bar{\alpha}}^{*} R_{\bar{\alpha}}+\sum_{\gamma \neq l} \sum_{l} R_{\bar{\alpha}}^{*} K_{\alpha j \bar{\alpha} k}^{\gamma l} \Phi_{\gamma l}
$$

Recall $R_{\bar{\alpha}} \in \mathcal{H}_{\alpha \bar{\alpha}}$ and $\Phi_{\alpha j} \Phi_{\bar{\alpha} k} \in \mathcal{H}_{\alpha \bar{\alpha}}$. Therefore we get

$$
R_{\bar{\alpha}}^{*} \Phi_{\alpha j} \Phi_{\bar{\alpha} k}=\left(R_{\bar{\alpha}}, \Phi_{\alpha j} \Phi_{\bar{\alpha} k}\right)_{\mathcal{H}_{\alpha \bar{\alpha}}}=\lambda_{j k} d(\alpha) 1+\sum_{\gamma \neq \iota} \sum_{l} R_{\bar{\alpha}}^{*} K_{\alpha j \bar{\alpha} k}^{\gamma l} \Phi_{\gamma l}
$$

Now we calculate

$$
\left(R_{\bar{\alpha}}, \Phi_{\alpha j} \Phi_{\bar{\alpha} k}\right)_{\mathcal{H}_{\alpha \bar{\alpha}}}=\left(J(\alpha, \bar{\alpha}) \sum_{l=1}^{d(\alpha)} \Phi_{\alpha l} \otimes \Phi_{\bar{\alpha} l}, J(\alpha, \bar{\alpha})\left(\Phi_{\alpha j} \otimes \Phi_{\bar{\alpha} k}\right)\right)_{\mathcal{H}_{\alpha \bar{\alpha}}}
$$




$$
=\sum_{l=1}^{d(\alpha)}\left(\Phi_{\alpha l}, \Phi_{\alpha j}\right)_{\mathcal{H}_{\alpha}}\left(\Phi_{\bar{\alpha} l}, \Phi_{\bar{\alpha} k}\right)_{\mathcal{H}_{\bar{\alpha}}}=\sum_{l=1}^{d(\alpha)} \delta_{l j} \delta_{l k}=\delta_{j k}
$$

and we obtain

$$
\delta_{j k} 1=\lambda_{j k} d(\alpha) 1+\sum_{\gamma \neq \iota} \sum_{l} R_{\bar{\alpha}}^{*} K_{\alpha j \bar{\alpha} k}^{\gamma l} \Phi_{\gamma l} .
$$

Since the system $\left\{\Phi_{\rho j}\right\}_{\rho j}$ forms an $\mathcal{A}$-leftmodule basis we finally get

$$
\lambda_{j k}=\frac{1}{d(\alpha)} \delta_{j k}
$$

and $(20)$ is proved for $\alpha=\beta$, too.

6.2.COROLLARY. The relation

$$
\left\langle X, Y F^{*}\right\rangle_{\mathcal{A}}=\langle X F, Y\rangle_{\mathcal{A}} ; \quad X, Y, F \in \mathcal{F}_{0}
$$

holds.

Proof. We already know that $\langle F, G\rangle_{\mathcal{A}}$ equals the $\mathcal{A}$-component of $F G^{*}$. Now

$$
X\left(Y F^{*}\right)^{*}=X F Y^{*}
$$

holds. Therefore their $\mathcal{A}$-components also coincide.

Next we introduce a $C^{*}$-norm on $\mathcal{F}_{0}$. The corresponding arguments are standard and follow the lines of Doplicher/Roberts, e.g. in [1,p.88 f.]; see also [7,p.217 f.] and $[7, p .286$ f. $]$.

The first step is to introduce a norm in $\mathcal{F}_{0}$ by the definition

$$
|F|_{\mathcal{A}}:=\left\|\langle F, F\rangle_{\mathcal{A}}\right\|^{1 / 2}
$$

Obviously $|A|_{\mathcal{A}}=\|A\|$ for $A \in \mathcal{A}$ because $\left\|A A^{*}\right\|^{1 / 2}=\|A\|$. Note that $|\cdot|_{\mathcal{A}}$ fails to be a $C^{*}$-norm. Therefore in a second step we define the following norm on $\mathcal{F}_{0}$ :

$$
\|F\|_{*}:=|\pi(F)|, \quad F \in \mathcal{F}_{0},
$$

where $(\pi(F)) X:=X F^{*}, X \in \mathcal{F}_{0}$ and $|\pi(F)|$ means the operator norm w.r.t. the norm $|\cdot|_{\mathcal{A}}$ on $\mathcal{F}_{0}$, i.e..

$$
|\pi(F)|=\sup _{|X|_{\mathcal{A}} \leq 1}\left|X F^{* *}\right|_{\mathcal{A}} .
$$

$\|\cdot\|_{*}$ turns out to be a $C^{*}$-norm on $\mathcal{F}_{0}$. Note that the $C^{*}$-property of $\|\cdot\|_{*}$ follows from Corollary 6.2. which says that

$$
\langle X, \pi(F) Y\rangle_{\mathcal{A}}=\left\langle\pi\left(F^{*}\right) X, Y\right\rangle_{\mathcal{A}}, \quad X, Y, F \in \mathcal{F}_{0}
$$

Note further that $\|\cdot\|_{*}$ on $\mathcal{F}_{0}$ is an extension of the $C^{*}$-norm $\|\cdot\|$ on $\mathcal{A} \subset \mathcal{F}_{0}$, i.e.

$$
\|A\|_{*}=\|A\|, \quad A \in \mathcal{A} .
$$

Moreover, we have

$$
|F|_{\mathcal{A}} \leq\|F\|_{*}, \quad F \in \mathcal{F}_{0} .
$$


Finally we form the $C^{*}$-algebra

$$
\mathcal{F}:=\operatorname{clo}_{\|\cdot\| *}\left(\mathcal{F}_{0}\right)
$$

Now we turn to a special automorphism group $\mathcal{G}$ of $\mathcal{F}$. First we consider again the *subalgebra $\mathcal{F}_{0} \subseteq \mathcal{F}$ which is dense in $\mathcal{F}$ and consider automorphisms $g$ of $\mathcal{F}_{0}$ requiring invariance of the $\mathcal{A}$-scalar product $\langle\cdot, \cdot\rangle_{\mathcal{A}}$ :

6.3.DEFINITION. The automorphism $g \in$ aut $\mathcal{F}_{0}$ is said to be a symmetry if

$$
\left\langle g F_{1}, g F_{2}\right\rangle_{\mathcal{A}}=\left\langle F_{1}, F_{2}\right\rangle_{\mathcal{A}}, \quad F_{1}, F_{2} \in \mathcal{F}_{0}
$$

The collection of all automorphisms $g \in$ aut $\mathcal{F}_{0}$ of this type forms a group $\mathcal{G} \neq \emptyset$, because the identical automorphism id: id $F:=F$ belongs to $\mathcal{G}$. The group $\mathcal{G}$ is called the symmetry group.

6.4.LEMMA. Each automorphism $g \in \mathcal{G}$ can be uniquely extended to an automorphism of $\mathcal{F}$ so that $\mathcal{G}$ can be considered as a subgroup of aut $\mathcal{F}, \quad \mathcal{G} \subseteq$ aut $\mathcal{F}$.

Proof. From (22) and (24) it follows immediately that $|g F|_{\mathcal{A}}=|F|_{\mathcal{A}}, F \in \mathcal{F}_{0}$. Furthermore, we have $\|g F\|_{*}=\|F\|_{*}$ for all $F \in \mathcal{F}_{0}$ because from (23) we obtain

$$
\begin{gathered}
\|g F\|_{*}=|\pi(g F)|=\sup _{|X|_{\mathcal{A}} \leq 1}\left|X(g F)^{*}\right|_{\mathcal{A}}=\sup _{|X|_{\mathcal{A}} \leq 1}\left|g\left\{\left(g^{-1} X\right) F^{*}\right\}\right|_{\mathcal{A}} \\
=\sup _{|X|_{\mathcal{A}} \leq 1}\left|\left(g^{-1} X\right) F^{*}\right|_{\mathcal{A}}=\sup _{|Y|_{\mathcal{A}} \leq 1}\left|Y F^{*}\right|_{\mathcal{A}}=|\pi(F)|=\|F\|_{*} .
\end{gathered}
$$

Therefore, by continuous (isometric) extension each $g$ can be extended to the whole $C^{*}$-algebra $\mathcal{F}$, yielding an automorphism of $\mathcal{F}$.

We emphasize that the symmetry group $\mathcal{G}$ coincides with the stability group stab $\mathcal{A} \subset$ aut $\mathcal{F}$ of $\mathcal{F}$. Recall that stab $\mathcal{A}:=\{g \in$ aut $\mathcal{F}: g A=A$ for all $A \in \mathcal{A}\}$.

First we have

6.5.LEMMA. Each Hilbert space $\mathcal{H}_{\alpha}, \alpha \in \mathcal{T}$, is invariant w.r.t. $g \in \operatorname{stab} \mathcal{A}$.

Proof. Recall that $X \in \mathcal{H}_{\alpha}$ iff $\alpha(A) X=X A$ for all $A \in \mathcal{A}$. Therefore we have

$$
\alpha(A) \cdot g X=g X \cdot A, \quad X \in \mathcal{H}_{\alpha}, A \in \mathcal{A}, g \in \operatorname{stab} \mathcal{A},
$$

i.e. $g X \in \mathcal{H}_{\alpha}$.

Now we prove

6.6.LEMMA. The relation

$$
\mathcal{G}=\operatorname{stab} \mathcal{A}
$$

holds.

Proof. First we prove $\mathcal{G} \subseteq \operatorname{stab} \mathcal{A}$. Let $A \in \mathcal{A}$. Then we have to prove that $g A=A$ for all $g \in \mathcal{G}$. Note that a priori $g A \in \mathcal{F}_{0}$. We put $\mathcal{F}_{0} \ni F=\sum_{\alpha, j}(F)_{\alpha j} \Phi_{\alpha j}$ with fixed orthonormal bases $\left\{\Phi_{\alpha j}\right\}_{j=1}^{d(\alpha)}$. For example $(F)_{l}$ is the $\mathcal{A}$-component of $F$. Recall that $\langle F, 1\rangle_{\mathcal{A}}=(F)_{\text {,. Then }}$

$$
\left\langle g A, \Phi_{\alpha j}\right\rangle_{\mathcal{A}}=\left\langle A, g^{-1} \Phi_{\alpha j}\right\rangle_{\mathcal{A}}=A\left(g^{-1} \Phi_{\alpha j}\right)_{i}^{*}=0
$$


for $\alpha \neq \iota, j=1,2, \ldots, d(\alpha)$ because $g \mathcal{H}_{\alpha} \subseteq \mathcal{H}_{\alpha}$, i.e. $g A \in \mathcal{A}$. This implies

$$
g A=(g A)_{\imath}=\langle g A, 1\rangle_{\mathcal{A}}=\langle g A, g 1\rangle_{\mathcal{A}}=\langle A, 1\rangle_{\mathcal{A}}=A
$$

hence $g A=A$ follows.

Second we prove $\operatorname{stab} \mathcal{A} \subseteq \mathcal{G}$. Let $g \in \operatorname{stab} \mathcal{A}$. From Lemma 6.5. we get

$$
g \Phi_{\alpha j}=\sum_{j^{\prime}} u_{j^{\prime} j}^{(\alpha)}(g) \Phi_{\alpha j^{\prime}}
$$

where $\left\{u_{j^{\prime} j}^{(\alpha)}(g)\right\}_{j^{\prime}, j=1}^{d(\alpha)}$ is a unitary $d(\alpha) \times d(\alpha)$-matrix because

$$
\left(g \Phi_{\alpha j}\right)^{*} g \Phi_{\alpha j^{\prime}}=g\left(\Phi_{\alpha j}^{*} \Phi_{\alpha j^{\prime}}\right)=g\left(\delta_{j j^{\prime}} 1\right)=\delta_{j j^{\prime}} 1 .
$$

Now we have

$$
\left\langle g F_{1}, g F_{2}\right\rangle_{\mathcal{A}}=\sum_{\rho, j, \sigma, k} A_{\rho j}\left\langle g \Phi_{\rho j}, g \Phi_{\sigma k}\right\rangle_{\mathcal{A}} B_{\sigma k}^{*}
$$

In the case $\rho \neq \sigma$ we have $\left\langle g \Phi_{p j}, g \Phi_{\sigma k}\right\rangle_{\mathcal{A}}=0=\left\langle\Phi_{\rho j}, \Phi_{\sigma k}\right\rangle_{\mathcal{A}}$. In the other case $\rho=\sigma$ we get

$$
\begin{gathered}
\left\langle g \Phi_{\rho j}, g \Phi_{\rho k}\right\rangle_{\mathcal{A}}=\left\langle\sum_{j^{\prime}} u_{j^{\prime} j} \Phi_{\rho j^{\prime}}, \sum_{k^{\prime}} u_{k^{\prime} k} \Phi_{\rho k^{\prime}}\right\rangle_{\mathcal{A}}=\sum_{j^{\prime} k^{\prime}} u_{j^{\prime} j} \bar{u}_{k^{\prime} k}\left\langle\Phi_{\rho j^{\prime}}, \Phi_{\rho k^{\prime}}\right\rangle_{\mathcal{A}} \\
=\sum_{j^{\prime} k^{\prime}} u_{j^{\prime} j} \bar{u}_{k^{\prime} k} \frac{1}{d(\rho)} \delta_{j^{\prime} k^{\prime}}=\frac{1}{d(\rho)} \sum_{k^{\prime}} u_{k^{\prime} j} \bar{u}_{k^{\prime} k}=\frac{1}{d(\rho)} \delta_{j k}=\left\langle\Phi_{\rho j}, \Phi_{\rho k}\right\rangle_{\mathcal{A}}
\end{gathered}
$$

hence $\left\langle g F_{1}, g F_{2}\right\rangle_{\mathcal{A}}=\left\langle F_{1}, F_{2}\right\rangle_{\mathcal{A}}$ follows.

Further it turns out that $\mathcal{G}$ is compact.

6.7.PROPOSITION. The symmetry group $\mathcal{G}$ is compact w.r.t. the pointwise norm topology.

Proof. The Hilbert spaces $\mathcal{H}_{\alpha}, \operatorname{dim} \mathcal{H}_{\alpha}=d(\alpha)$, are invariant w.r.t. $\mathcal{G}$ and they generate $\mathcal{F}$, together with $\mathcal{A}$. Therefore, according to Doplicher/Roberts $[8$, Lemma 3.2.,p.292] $\mathcal{G}$ is compact w.r.t. the pointwise norm topology.

6.8.PROPOSITION. Let $\mathcal{F}_{0} \ni F=\sum_{\rho, j} F_{\rho j} \Phi_{\rho j}$, where the system of orthonormal bases $\left\{\Phi_{\rho j}\right\}_{j=1}^{d(\rho)}$ is fixed. Then

$$
\left\|F_{\rho j}\right\| \leq d(\rho)\|F\|_{*}, \quad j=1,2, \ldots d(\rho), \rho \in \hat{\mathcal{T}},
$$

i.e. $F_{\rho j}$ depends continuously on $F$.

Proof. We have

$$
\frac{1}{d(\rho)}\left\|F_{\rho j}\right\|^{2}=\frac{1}{d(\rho)}\left\|F_{\rho j} F_{\rho j}^{*}\right\| \leq\left\|\sum_{\alpha, j} d(\alpha)^{-1} F_{\alpha j} F_{\alpha j}^{*}\right\|=\left\|\langle F, F\rangle_{\mathcal{A}}\right\|^{2}=|F|_{\mathcal{A}}^{2} \leq\|F\|_{*}^{2}
$$

This proves $(25)$.

6.9.COROLLARY. The $\mathcal{A}$-scalar product $\left\langle F_{1}, F_{2}\right\rangle_{\mathcal{A}}$ on $\mathcal{F}_{0}$ is continuous w.r.t. the $C^{*}$-norm $\|\cdot\|_{*}$. 
Proof. According to Lemma 6.1., we have $\left\langle F_{1}, F_{2}\right\rangle_{\mathcal{A}}=\left(F_{1} F_{2}^{*}\right)_{1}$. So it is sufficient to prove that $F$, depends continuously on $F$. But this assertion is a special case of Proposition 6.8 .

Corollary 6.9. implies that the $\mathcal{A}$-scalar product has a continuous extension to $\mathcal{F}$ w.r.t. $\|\cdot\|_{*}$.

Obviously, on $\mathcal{F}_{0}$ we can define projections $\Pi_{p}, \rho \in \hat{\mathcal{T}}$, by the definition

$$
\mathcal{F}_{0} \ni F^{\prime}=\sum_{\sigma, j} A_{\sigma j} \Phi_{\sigma j} \rightarrow \Pi_{\rho} F:=\sum_{j=1}^{d(\rho)} A_{\rho j} \Phi_{\rho j} \in \operatorname{spa}\left\{\mathcal{A} \mathcal{H}_{\rho}\right\}
$$

Note that this definition does not depend on the special orthonormal basis in $\mathcal{H}_{\rho}$.

$\Pi_{\rho} F$ depends continuously on $F$ w.r.t. $\|\cdot\|_{*}$, in particular we get

$$
\left\|\Pi_{\rho} F\right\| \leq d(\rho)^{2}\|F\|_{*}, \quad F \in \mathcal{F}_{0} .
$$

Therefore, $\Pi_{\rho}$ can be continuously extended on $\mathcal{F}$ as a projection and we obtain the relations

$$
\begin{gathered}
1 \leq\left\|\Pi_{\rho}\right\|_{*} \leq d(\rho)^{2}, \\
\operatorname{Il}_{\rho}\left(A_{1} F A_{2}\right)=A_{1} \Pi_{\rho}(F) A_{2}, \quad f \in \mathcal{F}, A_{1}, A_{2} \in \mathcal{A}, \\
\Pi_{\rho} \Pi_{\sigma}=\Pi_{\sigma} \Pi_{\rho}=\delta_{\rho \sigma} \Pi_{\rho},
\end{gathered}
$$

for a fixed system of representers $\rho, \sigma \in \hat{\mathcal{T}}$.

6.10.COROLLARY. The relative commutant $\mathcal{A}^{\prime} \cap \mathcal{F}$ is trivial, $\mathcal{A}^{\prime} \cap \mathcal{F}=\mathrm{C} 1$.

Proof. Let $F \in \mathcal{F}$ and $A F=F A$ for all $A \in \mathcal{A}$. Then

$$
A \cdot \Pi_{\rho} F=\Pi_{\rho}(A F)=\Pi_{\rho}(F A)=\Pi_{\rho} F \cdot A, \quad \rho \in \hat{\mathcal{T}} .
$$

This means $\Pi_{\rho} F \in \mathcal{A}^{\prime} \cap \mathcal{F}_{0}$, i.e. $\Pi_{\rho} F=\lambda_{\rho} 1$. hence for $\rho \neq \imath$ one has $\lambda_{\rho}=0$ and this implies $F=\lambda_{\iota} 1$.

6.11.LEMMA. The relation

$$
\Pi_{\rho} \mathcal{F}=\operatorname{spa}\left\{\mathcal{A} \mathcal{H}_{\rho}\right\}, \quad \rho \in \hat{\mathcal{T}}
$$

is valid.

Proof. Obviously, $\Pi_{\rho} \mathcal{F}_{0}=\operatorname{spa} \mathcal{A} \mathcal{H}_{\rho}$. Since $\Pi_{\rho} \mathcal{F}=\operatorname{clo}_{\| H_{*}} \Pi_{\rho} \mathcal{F}_{0}$ it is sufficient to prove that spa $\mathcal{A} \mathcal{H}_{\rho}$ is closed w.r.t. $\|\cdot\|_{*}$. Let $F_{n}=\sum_{j=1}^{d(\rho)} A_{\rho j}^{(n)} \Phi_{\rho j} \in$ spa $\mathcal{A H}_{\rho}$ and $\left\|F_{n}-F_{m}\right\|_{*} \rightarrow 0, n, m \rightarrow \infty$. The limit is denoted by $F_{\infty}$. Then from $\left|F_{n}-F_{m}\right|_{A}^{2} \leq$ $\left\|F_{n}-F_{m}\right\|_{*}^{2}$ we get

$$
\begin{gathered}
\left\|\left\langle F_{n}-F_{m}, F_{n}-F_{m}\right\rangle_{\mathcal{A}}\right\|=\left\|\sum_{j=1}^{d(\rho)}\left(A_{\rho j}^{(n)}-A_{\rho j}^{(m)}\right)\left(A_{\rho j}^{(n)}-A_{\rho j}^{(m)}\right)^{*}\right\| \\
\geq\left\|\left(A_{\rho j}^{(n)}-A_{\rho j}^{(m)}\right)\left(A_{\rho j}^{(n)}-A_{\rho j}^{(m)}\right)^{*}\right\|=\left\|A_{\rho j}^{(n)}-A_{\rho j}^{(m)}\right\|^{2} \rightarrow 0
\end{gathered}
$$

hence $F_{\infty}=\sum_{j=1}^{d(\rho)} A_{\rho j}^{\infty} \Phi_{\rho j}$ and $F_{\infty} \in \operatorname{spa} \mathcal{A} \mathcal{H}_{\rho}$. 
6.12.LEMMA. The projection $\Pi_{\rho}$ commutes with the action of $\mathcal{G}$, i.e.

$$
\Pi_{\rho}(g F)=g \Pi_{\rho} F, \quad F \in \mathcal{F}, g \in \mathcal{G} .
$$

Proof. It is sufficient to prove (26) for all elements $F \in \mathcal{F}_{0}$ because (26) then follows using extension by continuity. But since $\mathcal{H}_{\rho}$ is invariant w.r.t. $\mathcal{G}$ and $g A=A$ for all $A \in \mathcal{A}$, we have $g \sum_{\rho, j} A_{\rho j} \Phi_{\rho j}=\sum_{\rho, j} A_{\rho j} g \Phi_{\rho j}$, hence $g\left(\Pi_{\rho} F\right)=\sum_{j=1}^{d(\rho)} A_{\rho j} g \Phi_{\rho j}=$ $\Pi_{\rho}(g F)$ follows.

6.13.PROPOSITION. The fixed point algebra of $\mathcal{G}$ coincides with $\mathcal{A}$.

Proof. First we note that $U(\mathcal{K})$ is a subgroup of $\mathcal{G}, U(\mathcal{K}) \subseteq \mathcal{G}$. This is obvious because

$$
\left\langle U(k) F_{1}, U(k) F_{2}\right\rangle_{\mathcal{A}}=\left\langle F_{1}, F_{2}\right\rangle_{\mathcal{A}}, \quad k \in \mathcal{K}, F_{1}, F_{2} \in \mathcal{F} .
$$

Since $\mathcal{K}$ acts irreducibly on $\mathcal{H}_{\rho}, \rho \in \hat{\mathcal{T}}$, and $\mathcal{H}_{\rho}$ is invariant w.r.t. $\mathcal{G}$ it follows that $\mathcal{H}_{\rho}$ is irreducible w.r.t. $\mathcal{G}$, too. Now, according to Lemma 6.12 , from $g F=F$ for all $g \in \mathcal{G}$ we obtain $g \Pi_{\rho} F=\Pi_{\rho} F$, i.e. the $\Pi_{\rho}$-components of $F$ are also invariant. So we have to prove $\Pi_{\rho} F=0$ for all $\rho \neq \iota$. Now $\Pi_{\rho} F=\sum_{j=1}^{d(\rho)} A_{\rho j} \Phi_{\rho j}$ and $g\left(\Pi_{\rho} F\right)=$ $\sum_{j, j^{\prime}=1}^{d(\rho)} A_{\rho j} u_{j^{\prime} j}^{(\rho)}(g) \Phi_{\rho j^{\prime}}$ hence $A_{\rho j}=\sum_{j^{\prime}=1}^{d(\rho)} u_{j j^{\prime}}^{(\rho)}(g) A_{\rho j^{\prime}}$ follows. Integrating this over $\mathcal{G}$ we obtain $A_{\rho j}=0$.

6.14.COROLLARY. The groups $U(\mathcal{K})$ and $\mathcal{G}$ coincide.

Proof. First one has to prove that $\operatorname{spec} \mathcal{G}=\hat{\mathcal{G}}$, i.e. all irreducible representations of $\mathcal{G}$ occur. This is obtained by considering the scalar functions $I_{1}^{*} g\left(H_{2}\right), H_{1}, H_{2} \in$ $\mathcal{H}_{\alpha}, \alpha \in \mathcal{T}$. Using the Stone-Weierstra approximation theorem one gets that these functions span a dense set in $C(\mathcal{G})$ hence in $L^{2}(\mathcal{G})$. Then one can conclude that $U(\mathcal{K})=\mathcal{G}($ cf. Doplicher/Roberts $[8,3.3$. Theorem, p.293]).

We note that also a direct calculation shows that the representations induced by $\mathcal{G}$ on $\mathcal{H}_{\alpha}$ and $\mathcal{H}_{\bar{\alpha}}, \alpha \in \mathcal{T}$, are mutually conjugated. Namely we have $R_{\alpha}=\sum_{j=1}^{d(\alpha)} \Phi_{\bar{\alpha} j} \Phi_{\alpha j}$ hence $R_{\alpha}=\sum_{j=1}^{d(\alpha)}\left(g \Phi_{\bar{\alpha} j}\right)\left(g \Phi_{\alpha j}\right)$ and one obtains immediately

$$
u_{j^{\prime} j}^{(\bar{\alpha})}(g)=\overline{u_{j^{\prime} j}^{(\alpha)}(g)}, \quad g \in \mathcal{G} .
$$

Finally we note that the objects $\alpha \in \mathcal{T}$ turn out to be the restrictions to $\mathcal{A}$ of the so-called canonical endomorphisms w.r.t. $\mathcal{H}_{\alpha}$,

$$
\alpha(X)=\sum_{j=1}^{d(\alpha)} \Phi_{\alpha j} X \Phi_{\alpha j}^{*}, \quad X \in \mathcal{A}, \alpha \in \mathcal{T} .
$$

\section{Acknowledgment}

It is a pleasure to thank S.Doplicher for a discussion on the subject at Oberwolfach in February 1994. I also thank Fatma Kärsten who contributed by some useful remarks concerning this approach. 


\section{References}

[1] S.Doplicher and J.E.Roberts: Endomorphisms of $C^{*}$-algebras, cross products and duality for compact groups, Ann. Math. 130, 75-119 (1989)

[2] S.Doplicher and J.E.Roberts: A new duality theory for compact groups, Invent. Math. 98, 157-218 (1989)

[3] S.Doplicher and J.E.Roberts: Why there is a field algebra with a compact gauge group describing the superselection structure in particle physics, Commun. Math. Phys. 131, 51-107 (1990)

[4] S.Doplicher and J.E.Roberts: Compact Lie groups associated with endomorphisms of $C^{*}$-algebras, Bull. Amer. Math. Soc. 11, 333-338 (1984)

[5] S.Doplicher and J.E.Roberts: $C^{*}$-algebras and duality for compact groups: why there is a compact group of internal gauge symmetries in particle physics, in Proc. Intern. Conf. Math. Phys., pp. 489-498, World Scientific, Singapore (1986)

[6] H.Baumgärtel: On the Reconstruction Problem in Superselection 'Theory, Math. Nachr. 161, 361-367 (1993)

[7] H.Baumgärtel and M.Wollenberg: Causal Nets of Operator Algebras - Mathematical Aspects of Algebraic Quantum Field Theory, Akademie Verlag, Berlin (1992)

[8] S.Doplicher and J.E.Roberts: Compact group actions on $C^{*}$-algebras, J. Operator Theory 19, 283-305 (1988)

[9] R.V.Kadison and J.R.Ringrose: Fundamentals of the Theory of Operator Algebras II, Academic Press, New York (1986)

Anschrift des Verfassers:

Prof.H.Baumgärtel

Mathematisches Institut

Universität Potsdam

Am Neuen Palais 10

Postfach 601553

14415 Potsdam 
